\title{
Old dualities and new anomalies
}

\author{
Roberto Bonezzi®, ${ }^{*}$ Felipe Díaz-Jaramillo®, ${ }^{\dagger}$ and Olaf Hohm \\ Institute for Physics, Humboldt University Berlin, Zum Großen Windkanal 6, D-12489 Berlin, Germany
}

(Received 29 September 2020; accepted 19 October 2020; published 1 December 2020)

\begin{abstract}
We revisit the question whether the worldsheet theory of a string admits a global $O(d, d)$ symmetry. We consider the truncation of the target space theory in which fields are independent of $d$ coordinates, which is $O(d, d, \mathbb{R})$ invariant. The worldsheet theory is not $O(d, d, \mathbb{R})$ invariant, unless it is truncated by setting winding and center-of-mass momenta to zero. We prove consistency of this truncation and give a manifestly $O(d, d, \mathbb{R})$ invariant action, generalizing a formulation due to Tseytlin by including all external and internal target space fields. It is shown that, due to chiral bosons, this symmetry is anomalous. The anomaly is cancelled by a Green-Schwarz mechanism that utilizes the external $B$-field.
\end{abstract}

DOI: 10.1103/PhysRevD.102.126002

\section{INTRODUCTION}

T-duality is a property of string theory that emerges upon quantizing the string on a toroidal background. Naturally, there have been numerous papers addressing the question to which extent and in which sense the T-duality group $O(d, d)$, either in its discrete or continuous version, is a duality or symmetry of the (classical or quantum) worldsheet theory (see [1-18] for an incomplete list of references). We nevertheless come back to this issue, partly motivated by recent developments on the interplay of higher-derivative $\alpha^{\prime}$ corrections and $O(d, d, \mathbb{R})$ invariance of the target space theory, both in conventional $[19,20]$ and in double field theory formulations [21-26]. We will first identify a certain consistent truncation of the worldsheet theory of the bosonic string in which $O(d, d, \mathbb{R})$ is a manifest symmetry classically, and second argue that this symmetry becomes anomalous quantum mechanically due to the worldsheet scalars being chiral bosons. This in turn implies that a Green-Schwarz-type mechanism is required in close analogy to anomaly cancellation in heterotic string theory.

We begin by asking the following: Is the (classical or quantum) string worldsheet theory $O(d, d)$ invariant, either under the discrete or continuous group? The first relevant observation here is that in the Hamiltonian formulation of the worldsheet theory, for arbitrary backgrounds, a "generalized metric" $\mathcal{H}_{M N}$ emerges that combines the metric and $B$-field into an $O(d, d)$ matrix. However, as we will review, this does

\footnotetext{
*roberto.bonezzi@physik.hu-berlin.de felipe.diaz-jaramillo@hu-berlin.de

‡ohohm@physik.hu-berlin.de
}

Published by the American Physical Society under the terms of the Creative Commons Attribution 4.0 International license. Further distribution of this work must maintain attribution to the author(s) and the published article's title, journal citation, and DOI. Funded by SCOAP ${ }^{3}$. not mean that the worldsheet theory has a locally realized $O(d, d)$ symmetry in general. A genuine $O(d, d)$ duality invariance is usually only expected to emerge on toroidal backgrounds. Suppose then that the target space is a torus in which a classical string propagates. Is the worldsheet theory $O(d, d)$ invariant? It is not, because there are winding modes that are discrete, due to the topology of the torus, while the center-of-mass momenta, which should pair up with the winding modes into an $O(d, d)$ multiplet, are continuous. One could constrain the momenta by hand to be discrete, but there seems to be no physical justification for doing so. Similarly, one could promote the winding numbers to dynamical fields (functions of worldsheet coordinates), but then one is no longer dealing with a theory of strings since the worldsheet scalars are not well-defined maps on the torus preserving the torus boundary conditions. Rather, the proper $O(d, d, \mathbb{Z})$ emerges when quantizing the worldsheet theory, because then the momenta are quantized, hence naturally pairing up with the discrete winding numbers. More precisely, the $O(d, d, \mathbb{Z})$ is then a duality (T-duality) in which a change of background leads to a physically equivalent theory in which momentum and winding is exchanged.

The above is standard textbook folklore of string theory, but here we will revisit the issue from a slightly different point of view. We start from the observation that when one truncates the target space theory by taking all fields to be independent of $d$ coordinates (for instance, by restricting to the massless fields for Kaluza-Klein compactification on a torus $T^{d}$ ) a global $O(d, d, \mathbb{R})$ symmetry emerges. ${ }^{1}$ If we now couple a classical string to this theory (in the same way

\footnotetext{
${ }^{1}$ This symmetry is in fact a consequence of the $O(d, d, \mathbb{Z})$ duality that target space closed string theory exhibits, since in the truncation both the usual massive Kaluza-Klein modes and their dual "winding" Kaluza-Klein modes disappear, so that the theory loses all memory of the torus topology, thereby enhancing $O(d, d, \mathbb{Z})$ to $O(d, d, \mathbb{R})$.
} 
that one may couple a point particle to Einstein gravity) does the combined system have an $O(d, d, \mathbb{R})$ symmetry? We will show that in general it does not, not even under the discrete subgroup, but that there is such a symmetry if one truncates also the worldsheet theory by setting winding and center-of-mass momenta to zero. This makes sense since it reflects the truncation of the target space theory. Note that this truncation only refers to the internal sector (the coordinate directions on which the target space fields no longer depend), and so the string still has a nontrivial dynamics thanks to the external space. As one of our technical results we establish consistency of this truncation, for which the worldsheet action takes the manifestly $O(d, d, \mathbb{R})$ invariant form

$$
\begin{aligned}
S= & -\frac{1}{4 \pi \alpha^{\prime}} \int d^{2} \sigma\left[\sqrt{-h} h^{\alpha \beta} g_{\mu \nu} \partial_{\alpha} X^{\mu} \partial_{\beta} X^{\nu}\right. \\
& \left.+\epsilon^{\alpha \beta}\left(B_{\mu \nu} \partial_{\alpha} X^{\mu} \partial_{\beta} X^{\nu}-\mathcal{A}_{\mu}{ }^{M} \partial_{\alpha} Y_{M} \partial_{\beta} X^{\mu}\right)\right] \\
& +\frac{1}{4 \pi \alpha^{\prime}} \int d^{2} \sigma\left[D_{\sigma} Y^{M} D_{\tau} Y_{M}-u D_{\sigma} Y^{M} D_{\sigma} Y_{M}\right. \\
& \left.-e \mathcal{H}_{M N} D_{\sigma} Y^{M} D_{\sigma} Y^{N}\right],
\end{aligned}
$$

where $X^{\mu}$ and $Y^{M}$ are the embedding scalars for the external and internal space, respectively, and $g_{\mu \nu}, B_{\mu \nu}, \mathcal{A}_{\mu}{ }^{M}$ and $\mathcal{H}_{M N}$ are target space fields depending only on $X$. Moreover, we defined the covariant derivative

$$
D_{\alpha} Y^{M}:=\partial_{\alpha} Y^{M}+\mathcal{A}_{\mu}{ }^{M}(X) \partial_{\alpha} X^{\mu}
$$

with worldsheet coordinates $\sigma^{\alpha}=(\tau, \sigma)$. This action is manifestly $O(d, d, \mathbb{R})$ invariant, with $M, N=1, \ldots, 2 d$ being fundamental $O(d, d, \mathbb{R})$ indices. In particular, the internal scalars $Y^{M}$ are doubled, but the above action is equivalent to the standard sigma model action in this truncation, since the second-order field equations imply, through integration and gauge fixing, first-order duality relations. The above action generalizes a reformulation of the worldsheet action due to Tseytlin [1,2] by including all external and internal target space fields that survive the truncation, in particular the external $B$-field that turns out to be instrumental for the Green-Schwarz mechanism. The above Lagrangian, which provides an action principle for equations of motion given by Maharana and Schwarz in [6], was also given by Schwarz and Sen in [27] and revisited recently in [28]. The action is indeed invariant under two-dimensional diffeomorphisms and Weyl transformations, albeit not manifestly so, since the worldsheet coordinates have been split in the second line, where $e$ and $u$ are the components of the worldsheet metric $h_{\alpha \beta}$, defined by $e=\sqrt{-h} h_{\sigma \sigma}^{-1}$ and $u=h_{\tau \sigma} h_{\sigma \sigma}^{-1}$. We will give a careful analysis of the nonmanifest two-dimensional diffeomorphism invariance and of the complete Virasoro constraints.

The results above apply to the classical worldsheet theory. As the second main point of this paper we then turn to its quantization and point out that since the worldsheet scalars are chiral (self-dual) bosons, the $O(d, d, \mathbb{R})$ symmetry of the classical theory is expected to be anomalous. More precisely, it is technically and conceptually easier to work in a frame formulation, based on the coset space $O(d, d, \mathbb{R}) / S O(d, \mathbb{R})_{L} \times S O(d, \mathbb{R})_{R}$, where it is the gauge group $S O(d, \mathbb{R})_{L} \times S O(d, \mathbb{R})_{R}$ that becomes anomalous. The presence of anomalies is confirmed independently by the recent result that in the target space theory the $O(d, d, \mathbb{R})$ symmetry, or alternatively the $S O(d, \mathbb{R})_{L} \times S O(d, \mathbb{R})_{R}$ symmetry, requires a deformation at order $\alpha^{\prime}[19,20]$, which from the point of view of the worldsheet theory cancels the anomaly via the GreenSchwarz mechanism. This state of affairs mimics heterotic string theory, for which the worldsheet theory is anomalous due to the presence of chiral fermions, which gives a worldsheet interpretation of the Green-Schwarz mechanism $[29,30]$. In the present context we prove that the one-loop effective action $W$ defined in terms of (1.1) by

$$
e^{i W[g, B, \mathcal{A}, E]}=Z^{-1} \int D Y e^{i S}
$$

transforms under $S O(d, \mathbb{R})_{L} \times S O(d, \mathbb{R})_{R}$ to lowest order as

$$
\delta_{\lambda} W=\frac{1}{8 \pi} \int \operatorname{tr}(d \lambda \wedge Q)-\frac{1}{8 \pi} \int \operatorname{tr}(d \bar{\lambda} \wedge \bar{Q}) .
$$

Here $E$ is a frame field for $\mathcal{H}_{M N}$ and $Q$ and $\bar{Q}$ are the (composite) $S O(d, \mathbb{R})_{L} \times S O(d, \mathbb{R})_{R}$ connections. This anomaly is cancelled by assigning the following transformation to the $B$-field:

$$
\delta_{\lambda} B_{\mu \nu}=\frac{\alpha^{\prime}}{2} \operatorname{tr}\left(\partial_{[\mu} \lambda Q_{\nu]}\right)-\frac{\alpha^{\prime}}{2} \operatorname{tr}\left(\partial_{[\mu} \bar{\lambda} \bar{Q}_{\nu]}\right) .
$$

We also discuss and establish various other features regarding the quantum consistency of the worldsheet theory, including absence of gravitational anomalies [31], see also [32,33].

This paper is organized as follows. In Sec. II we review and clarify the Hamiltonian formulation of the worldsheet theory for strings with a toroidal target space. In particular, we introduce the proper truncation in which $O(d, d, \mathbb{R})$ will be made a manifest symmetry of the classical action. In Sec. III we turn to the coupling of the worldsheet theory to the target space string theory and display the worldsheet action and equations of motion in a manifestly $O(d, d, \mathbb{R})$ invariant form. Then, in Sec. IV, we show the presence of anomalies and the need to invoke a Green-Schwarz mechanism. We close with a brief conclusion and outlook section. In two Appendixes we perform a careful analysis of the diffemorphism invariance of the worldsheet theory, which is no longer manifest, and show that there are no gravitational anomalies. 


\section{CLASSICAL STRING ON A TORUS}

In this section we study the dynamics of a classical closed string on a toroidal target space $T^{d}$, representing the compact part of a $D=d+n$ dimensional spacetime with $d$ Abelian isometries, and we shall focus for the moment on the dynamics along those directions alone. In particular, we consider the string coordinate embeddings $X^{i}(\sigma, \tau)$ coupled to background fields $G_{i j}$ and $B_{i j}$ in the Polyakov sigma model

$S=-\frac{1}{4 \pi \alpha^{\prime}} \int d^{2} \sigma\left[\sqrt{-h} h^{\alpha \beta} \partial_{\alpha} X^{i} \partial_{\beta} X^{j} G_{i j}+\epsilon^{\alpha \beta} \partial_{\alpha} X^{i} \partial_{\beta} X^{j} B_{i j}\right]$,

where the worldsheet metric $h_{\alpha \beta}$ has Minkowski signature $(-,+)$ and $\epsilon^{01}=-1$. The compact space arises upon identifying $x^{i} \sim x^{i}+2 \pi L^{i}$, where $L^{i}:=\sqrt{\alpha^{\prime}} w^{i}$ with integer winding numbers $w^{i} \in \mathbb{Z}$. Correspondingly, the allowed boundary conditions for the closed string worldsheet fields read (we use $\sigma \in[0,2 \pi]$ )

$\Delta X^{i}(\tau):=X^{i}(2 \pi, \tau)-X^{i}(0, \tau)=2 \pi L^{i}, \quad \Delta h_{\alpha \beta}(\tau)=0$.

The classical configuration space of the closed string is thus split by boundary conditions into the direct sum of disjoint topological sectors, labeled by the winding vector $L^{i}$. The noncompact case is covered by setting $L^{i}=0$. In view of the boundary conditions (2.2) one can separate the winding sector as

$X^{i}(\sigma, \tau)=L^{i} \sigma+\bar{X}^{i}(\sigma, \tau)=L^{i} \sigma+\sum_{n \in \mathbb{Z}} x_{n}^{i}(\tau) e^{i n \sigma}$,

since the shifted field obeys $\Delta \bar{X}^{i}=0$. Let us mention that the variational principle with the action (2.1) is well defined, since neither $h_{\alpha \beta}, \partial_{\alpha} X^{i}$ nor $\delta X^{i}$ have winding contributions.

\section{A. Hamiltonian formulation}

We now turn to the Hamiltonian formulation, which turns out to be useful for identifying the symmetries. The first step is to find the momenta conjugate to $X^{i}$ :

$$
P_{i}=\frac{1}{2 \pi \alpha^{\prime}}\left[\frac{1}{e} G_{i j}\left(\dot{X}^{j}-u X^{j^{\prime}}\right)+B_{i j} X^{j^{\prime}}\right],
$$

where, as usual, a dot (prime) denotes a derivative with respect to $\tau(\sigma)$, and we defined the components of the worldsheet metric (that will become Hamiltonian Lagrange multipliers) via $h_{\alpha \beta}=\frac{\Omega}{e}\left(\begin{array}{cc}u^{2}-e^{2} & u \\ u & 1\end{array}\right), \quad h^{\alpha \beta}=\frac{1}{e \Omega}\left(\begin{array}{cc}-1 & u \\ u & e^{2}-u^{2}\end{array}\right)$.

The total Hamiltonian consists entirely of first class constraints, as it should be in any diffeomorphism invariant theory. The action can then be written as

$$
S=\int d^{2} \sigma\left[P_{i} \dot{X}^{i}-e \mathcal{H}-u \mathcal{N}\right],
$$

where

$$
\begin{aligned}
\mathcal{N}:= & P_{i} X^{i^{\prime}} \\
\mathcal{H}:= & \frac{1}{2}\left[2 \pi \alpha^{\prime} G^{i j} P_{i} P_{j}-2 G^{i k} B_{k j} P_{i} X^{j^{\prime}}\right. \\
& \left.+\frac{1}{2 \pi \alpha^{\prime}}\left(G-B G^{-1} B\right)_{i j} X^{i^{\prime}} X^{j^{\prime}}\right] .
\end{aligned}
$$

Naturally, upon integrating out $P_{i}$ by solving its own equations of motion and back-substituting into the action one recovers the Polyakov action (2.1). The functions in (2.7) are phase space constraints (also referred to as Virasoro constraints) that are the canonical generators of worldsheet diffeomorphisms, being the Hamiltonian counterparts of the traceless worldsheet stress-energy tensor $T_{\alpha \beta}$. We notice that the third degree of freedom of the metric $h_{\alpha \beta}$, its overall conformal factor $\Omega$, drops out of the action. In Hamiltonian language, conformal gauge corresponds to gauge fixing $e=1$ and $u=0$.

Both Hamiltonian constraints can be put in a formally $O(d, d)$ invariant form by defining

$$
Z^{M}:=\left(\begin{array}{c}
\partial_{\sigma} X^{i} \\
2 \pi \alpha^{\prime} P_{i}
\end{array}\right)
$$

as well as the $O(d, d)$ invariant metric $\eta_{M N}$ and the generalized metric $\mathcal{H}_{M N}$,

$\eta_{M N}=\left(\begin{array}{cc}0 & \delta_{i}{ }^{j} \\ \delta^{i}{ }_{j} & 0\end{array}\right), \quad \mathcal{H}_{M N}=\left(\begin{array}{cc}\left(G-B G^{-1} B\right)_{i j} & B_{i k} G^{k j} \\ -G^{i k} B_{k j} & G^{i j}\end{array}\right)$.

The functions in (2.7) can now be written as

$\mathcal{N}=\frac{1}{4 \pi \alpha^{\prime}} \eta_{M N} Z^{M} Z^{N}, \quad \mathcal{H}=\frac{1}{4 \pi \alpha^{\prime}} \mathcal{H}_{M N} Z^{M} Z^{N}$.

Although the Hamiltonian thus takes a formally $O(d, d)$ invariant form, the full action is not obviously $O(d, d)$ invariant. First, the $O(d, d)$ vector $Z^{M}$ in (2.8) is defined in terms of derivatives of the fundamental field $X^{i}$ and hence it is not clear whether there is a locally realized $O(d, d)$ 
symmetry even for the Hamiltonian terms. Second, the "symplectic current" $P_{i} \dot{X}^{i}$ is part of the action and not manifestly invariant.

In order to elucidate the above issues, we will work explicitly with Fourier modes defined as

$$
\begin{aligned}
X^{i}(\sigma, \tau) & =L^{i} \sigma+\sum_{n \in \mathbb{Z}} x_{n}^{i}(\tau) e^{i n \sigma}, \quad P_{i}(\sigma, \tau)=\sum_{n \in \mathbb{Z}} p_{i n}(\tau) e^{i n \sigma}, \\
e(\sigma, \tau) & =\sum_{n \in \mathbb{Z}} e_{n}(\tau) e^{i n \sigma}, \quad u(\sigma, \tau)=\sum_{n \in \mathbb{Z}} u_{n}(\tau) e^{i n \sigma}, \quad(2.11)
\end{aligned}
$$

where we recalled (2.3). Here we assume the reality conditions $\varphi_{n}^{*}=\varphi_{-n}$ for all modes $\varphi_{n}:=\left(x_{n}^{i}, p_{i n}, e_{n}, u_{n}\right)$. The action (2.6) then reads

$$
S=2 \pi \int d \tau \sum_{n \in \mathbb{Z}}\left[p_{i n} \dot{x}_{-n}^{i}-e_{-n} \mathcal{H}_{n}-u_{-n} \mathcal{N}_{n}\right]
$$

Before giving the explicit expression for the modes $\mathcal{H}_{n}$ and $\mathcal{N}_{n}$ of the Virasoro constraints, let us introduce Fourier modes for the $O(d, d)$ vector $Z^{M}$ as

$$
\begin{aligned}
Z^{M}(\sigma, \tau) & =L^{M}(\tau)+\sum_{n \neq 0} Z_{n}^{M}(\tau) e^{i n \sigma}, \text { where } \\
Z_{n}^{M}(\tau) & =\left(\begin{array}{c}
i n x_{n}^{i}(\tau) \\
2 \pi \alpha^{\prime} p_{i n}(\tau)
\end{array}\right), \quad n \neq 0, \\
L^{M}(\tau) & =\left(\begin{array}{c}
L^{i} \\
2 \pi \alpha^{\prime} p_{i 0}(\tau)
\end{array}\right) .
\end{aligned}
$$

At this point one can perform an invertible field redefinition by setting

$$
2 \pi \alpha^{\prime} p_{i n}(\tau)=: \operatorname{in} \tilde{x}_{i n}(\tau), \quad n \neq 0,
$$

that corresponds to defining dual coordinates $\tilde{X}_{i}(\sigma, \tau)$ via [1]

$$
2 \pi \alpha^{\prime} P_{i}=\partial_{\sigma} \tilde{X}_{i}
$$

Integration of (2.15) gives

$\tilde{X}_{i}(\sigma, \tau)=2 \pi \alpha^{\prime} p_{i 0}(\tau) \sigma+\tilde{x}_{i 0}(\tau)+\sum_{n \neq 0} \tilde{x}_{i n}(\tau) e^{i n \sigma}$,

which introduces a zero mode $\tilde{x}_{i 0}$ of $\tilde{X}_{i}$ that does not appear in the original action, and for which it is not clear that it can become part of an $O(d, d)$ multiplet. Moreover, note that the dual fields $\tilde{X}_{i}$ do not describe a closed string winding around a "dual torus," since their boundary conditions are not constant nor labeled by integers, and so in general the $\tilde{X}_{i}$ cannot combine with the $X^{i}$ into an irreducible $O(d, d)$ representation. Nonetheless, from (2.13) and (2.14) one can still define the $O(d, d)$ nonzero modes

$$
X_{n}^{M}(\tau):=\left(\begin{array}{c}
x_{n}^{i}(\tau) \\
\tilde{x}_{i n}(\tau)
\end{array}\right), \quad n \neq 0,
$$

and $L^{M}(\tau)$ as in (2.13).

Next, we rewrite the Virasoro constraints in terms of these Fourier modes. While the general Hamiltonian form of the action given above is valid for arbitrary backgrounds $G_{i j}$ and $B_{i j}$, we here focus on the torus and assume that the backgrounds are constant. The modes of the Virasoro constraints then take the formally $O(d, d)$ covariant form

$\mathcal{N}_{n}=\frac{\eta_{M N}}{4 \pi \alpha^{\prime}}\left[2 i n X_{n}^{M} L^{N}-\sum_{k} k(n-k) X_{k}^{M} X_{n-k}^{N}\right], \quad n \neq 0$,

$\mathcal{N}_{0}=\frac{\eta_{M N}}{4 \pi \alpha^{\prime}}\left[L^{M} L^{N}+\sum_{k} k^{2} X_{k}^{M} X_{-k}^{N}\right]$,

$\mathcal{H}_{n}=\frac{\mathcal{H}_{M N}}{4 \pi \alpha^{\prime}}\left[2 i n X_{n}^{M} L^{N}-\sum_{k} k(n-k) X_{k}^{M} X_{n-k}^{N}\right], \quad n \neq 0$,

$\mathcal{H}_{0}=\frac{\mathcal{H}_{M N}}{4 \pi \alpha^{\prime}}\left[L^{M} L^{N}+\sum_{k} k^{2} X_{k}^{M} X_{-k}^{N}\right]$,

while the symplectic term can be recast in the form

$$
2 \pi \int d \tau\left[p_{i 0} \dot{x}_{0}^{i}+\frac{1}{4 \pi \alpha^{\prime}} \eta_{M N} \sum_{n \neq 0} i n X_{n}^{M} \dot{X}_{-n}^{N}\right] .
$$

Even though the nonzero modes $X_{n}^{M}$ can transform under an arbitrary $O(d, d)$ rotation as $X_{n}^{\prime M}=\Omega^{M}{ }_{N} X_{n}^{N}$, the momentum-winding vector $L^{M}$ in general breaks the boundary conditions under $O(d, d)$ : if we parametrize the $\Omega$ matrix as

$$
\Omega^{M}{ }_{N}=\left(\begin{array}{cc}
\omega_{j}^{i} & \alpha^{i j} \\
\beta_{i j} & \gamma_{i}^{j}
\end{array}\right),
$$

one has $L^{\prime i}=\omega^{i}{ }_{j} L^{j}+2 \pi \alpha^{\prime} \alpha^{i j} p_{j 0}(\tau)$. The $\alpha$ transformation in particular yields a nonacceptable boundary condition for the duality-rotated coordinate $X^{\prime i}$. Even if one is only interested in transforming classical solutions, where $p_{i 0}(\tau)=k_{i}$ is constant, the discreteness of $L^{i}$, that descends purely from topology, is violated by $k_{i} \in \mathbb{R}^{d}$ for a general $O(d, d)$ rotation, even in the discrete subgroup $O(d, d, \mathbb{Z})$. As mentioned in the Introduction, one could truncate the spectrum by hand by taking the components $p_{i 0}=k_{i}$ to be integers, thus mimicking the quantization condition that, however, is not part of the original classical theory. In contrast, at the quantum level the eigenvalues of the momentum zero mode $\hat{p}_{i 0}$ take the discrete values $\frac{n_{i}}{2 \pi \sqrt{\alpha^{\prime}}}$, and so it is only here that one obtains the well-known T-duality group $O(d, d, \mathbb{Z})$.

It seems thus that neither the classical action nor the classical solutions of the closed string are invariant under $O(d, d)$. However, one can try to focus on a particular 
subsector of the classical theory: to begin with, we shall restrict the analysis to the topological sector with zero winding, i.e., $L^{i}=0$. Classical solutions are also labeled by a constant center-of-mass momentum $p_{i 0}=k_{i}$, and we will restrict our discussion to the class of zero momentum solutions, $p_{i 0}=0$. Clearly, this subclass of solutions is closed under continuous $O(d, d, \mathbb{R})$ rotations, since $L^{M}=0$ is invariant.

Some comments are now in order: first of all, restricting to string solutions with vanishing center-of-mass momentum looks quite unphysical. However, one has to keep in mind that in the full theory (to which we shall turn in the next section) the compact space is only part of the entire spacetime. In fact, the low-energy effective field theory, that displays the $O(d, d, \mathbb{R})$ symmetry, precisely consists of fields that do not probe the internal space. In particular, they have zero Kaluza-Klein momenta and, obviously, cannot display winding. In this respect, it seems natural to consider the string dynamics restricted to zero winding and internal momentum as the suitable probe for the low-energy spacetime field theory.

\section{B. Truncated dynamics and consistency}

In order to restrict the space of classical solutions to zero winding and center-of-mass momentum, one can consider the truncated action obtained by setting $L^{i}=0$ and $p_{i 0}=0$ in (2.12):

$S^{\prime}=2 \pi \int d \tau \sum_{n \in \mathbb{Z}}\left[\frac{1}{4 \pi \alpha^{\prime}} i n X_{n}^{M} \dot{X}_{-n M}-e_{-n} \mathcal{H}_{n}-u_{-n} \mathcal{N}_{n}\right]$,

with truncated Virasoro modes

$$
\begin{aligned}
& \mathcal{N}_{n}=-\frac{\eta_{M N}}{4 \pi \alpha^{\prime}} \sum_{k \in \mathbb{Z}} k(n-k) X_{k}^{M} X_{n-k}^{N}, \\
& \mathcal{H}_{n}=-\frac{\mathcal{H}_{M N}}{4 \pi \alpha^{\prime}} \sum_{k \in \mathbb{Z}} k(n-k) X_{k}^{M} X_{n-k}^{N} .
\end{aligned}
$$

The above action, which is manifestly $O(d, d, \mathbb{R})$ invariant, can be rewritten in local form as

$$
\begin{aligned}
S^{\prime}= & \frac{1}{4 \pi \alpha^{\prime}} \int d^{2} \sigma\left[\partial_{\sigma} X^{M} \partial_{\tau} X_{M}-e \mathcal{H}_{M N} \partial_{\sigma} X^{M} \partial_{\sigma} X^{N}\right. \\
& \left.-u \partial_{\sigma} X^{M} \partial_{\sigma} X_{M}\right] .
\end{aligned}
$$

This is Tseytlin's original proposal, but with twodimensional diffeomorphism invariance left intact (albeit in a nonmanifest form). Taking $X^{M}(2 \pi, \tau)=X^{M}(0, \tau)$ automatically sets both winding and center-of-mass momentum to zero, since $2 \pi \alpha^{\prime} p_{i 0}=\frac{1}{2 \pi} \int_{0}^{2 \pi} d \sigma \partial_{\sigma} \tilde{X}_{i}=0$.

The equations of motion for the nonzero modes $X_{n}^{M}$ (or, which is the same, $x_{n}^{i}$ and $p_{\text {in }}$ ) as well as the Virasoro constraints coincide with the original ones obtained from (2.6) or (2.12) upon choosing the solution $p_{i 0}=0$. On the other hand, one has to be more careful with the zero modes $X_{0}^{M}$ : neither $x_{0}^{i}$ nor $\tilde{x}_{i 0}$ appear in the action (2.23), that indeed has the obvious gauge symmetry $\delta X^{M}=\Xi^{M}(\tau)$. While this is fine for $\tilde{x}_{i 0}$, for which it is just a redundancy of the field redefinition $2 \pi \alpha^{\prime} P_{i}=\partial_{\sigma} \tilde{X}_{i}$, it is not equivalent for $x_{0}^{i}$ that does possess a nontrivial equation of motion in the original theory. We view the reduced action (2.23) as providing the dynamics for the nonzero modes $X_{n}^{M}$ and then establish that this is a consistent truncation of the full worldsheet theory. To this end we have to show that once a solution is provided for the nonzero modes (modulo worldsheet diffeomorphisms), we can embed it into a solution of the full theory. This means that we have to give $x_{0}^{i}$ in terms of the untruncated fields so that the complete equations of motion of the original theory are satisfied.

Let us then study the original field equation for the zero mode $x_{0}^{i}(\tau)$. Generally, the second-order Lagrangian equations of motion are equivalent to the two sets of Hamiltionian equations obtained by varying with respect to $P_{i}$ and $X^{i}$, respectively. The former equation can be obtained by inverting the definition of canonical momenta (2.4),

$$
\dot{X}^{i}=u X^{j \prime}+e G^{i j}\left(2 \pi \alpha^{\prime} P_{j}-B_{j k} X^{k \prime}\right) .
$$

The equation for the zero mode $x_{0}^{i}(\tau)$ can then be obtained by integrating over $\sigma$,

$$
\dot{x}_{0}^{i}(\tau)=V^{i}(\tau),
$$

where from (2.24) we notice that $V^{i}$ is naturally the upper component of the $O(d, d)$ vector

$$
V^{M}(\tau)=\left(\begin{array}{c}
V^{i}(\tau) \\
\tilde{V}_{i}(\tau)
\end{array}\right):=\frac{1}{2 \pi} \int_{0}^{2 \pi} d \sigma\left[u \partial_{\sigma} X^{M}+e \mathcal{H}^{M N} \partial_{\sigma} X_{N}\right]
$$

The Hamiltonian equation obtained by varying with respect to $X^{i}$ reduces for the zero modes to $\dot{p}_{i 0}=0$, since the functions (2.7) are independent of $x_{0}^{i}$ (they depend only on $\left.X^{i \prime}\right)$. Thus, these equations are trivially satisfied for $p_{i 0}=0$, and so we only have to worry about Eq. (2.25). Given a solution to the field equations derived from (2.23) (that leave the zero modes completely undetermined), one can directly integrate (2.25):

$$
x_{0}^{i}(\tau)=x^{i}+\int_{0}^{\tau} d \tau^{\prime} V^{i}\left(\tau^{\prime}\right) .
$$

This is the embedding into the full theory, which by construction satisfies the equations of motion. Note that 
we could use the lower component of (2.26) to similarly define a function $\tilde{x}_{i 0}(\tau)$, but there is no need to do so since such a dynamical variable does not appear in the original theory.

\section{GENERAL WORLDSHEET ACTION}

\section{A. Kaluza-Klein split}

In this section we are going to consider the more general sigma model of a closed string propagating on a target $(D=d+n)$-dimensional spacetime characterized by $d$ Abelian isometries. We will choose coordinates $\hat{x}^{\hat{\mu}}=\left(x^{\mu}, y^{i}\right)$, with $\mu=0, \ldots, n-1$ and $i=1, \ldots, d$ such that all spacetime fields are independent of $y^{i}$, being the isometry directions, either compact or not. The $n$-dimensional spacetime field content consists of [6] the following:

(i) the $n$-dimensional metric, dilaton and Kalb-Ramond fields $g_{\mu \nu}, \phi$ and $B_{\mu \nu}$,

(ii) $2 d$ Abelian gauge fields forming an $O(d, d)$ vector:

$$
\mathcal{A}_{\mu}{ }^{M}=\left(\begin{array}{c}
A_{\mu}^{i} \\
\tilde{A}_{\mu i}
\end{array}\right)
$$

which originate from the off-diagonal components of the higher dimensional metric $\hat{G}_{\hat{\mu} \hat{\nu}}$ and $B$ field $\hat{B}_{\hat{\mu} \hat{\nu}}$,

(iii) $d^{2}$ scalar fields $G_{i j}$ and $B_{i j}$ originating from the internal components of $\hat{G}_{\hat{\mu} \hat{\nu}}$ and $\hat{B}_{\hat{\mu} \hat{\nu}}$, that organize into the $O(d, d)$ valued generalized metric $\mathcal{H}_{M N}$.

The reduced $n$-dimensional effective field theory action reads [6]

$$
\begin{aligned}
S_{\mathrm{FT}} & =\frac{1}{2 \kappa^{2}} \int d^{n} x \sqrt{-g} e^{-2 \phi}\left[R+4 \partial_{\mu} \phi \partial^{\mu} \phi-\frac{1}{12} H_{\mu \nu \rho} H^{\mu \nu \rho}\right. \\
& \left.-\frac{1}{4} \mathcal{H}_{M N} \mathcal{F}_{\mu \nu}{ }^{M} \mathcal{F}^{\mu \nu N}+\frac{1}{8} \partial_{\mu} \mathcal{H}_{M N} \partial^{\mu} \mathcal{H}^{M N}\right]
\end{aligned}
$$

where $n$-dimensional spacetime indices are raised with the inverse metric $g^{\mu \nu}$. The Abelian field strength is given by

$$
\mathcal{F}_{\mu \nu}{ }^{M}=\partial_{\mu} \mathcal{A}_{\nu}{ }^{M}-\partial_{\nu} \mathcal{A}_{\mu}{ }^{M},
$$

while the three-form curvature $H_{\mu \nu \rho}$ needs an Abelian Chern-Simons modification compared to the naive form $H=d B$ :

$$
H_{\mu \nu \rho}:=3 \partial_{[\mu} B_{\nu \rho]}-3 \mathcal{A}_{[\mu}{ }^{M} \partial_{\nu} \mathcal{A}_{\rho] M},
$$

where the $O(d, d)$ indices have been contracted with the invariant metric $\eta_{M N}$. The effective action (3.2) is invariant under $n$-dimensional diffeomorphisms, as well as two-form gauge transformations $\delta_{\zeta} B_{\mu \nu}=\partial_{\mu} \zeta_{\nu}-\partial_{\nu} \zeta_{\mu}$. Invariance under the $U(1)^{2 d}$ gauge transformations $\delta_{\lambda} \mathcal{A}_{\mu}{ }^{M}=\partial_{\mu} \lambda^{M}$ requires the additional transformation of the $B$-field $\delta_{\lambda} B_{\mu \nu}=\frac{1}{2} \mathcal{F}_{\mu \nu}{ }^{M} \lambda_{M}$.

The sigma model describing the coupling of the string to the spacetime fields is most easily written in terms of the $(n+d)$-dimensional field content as ${ }^{2}$

$$
\begin{aligned}
S_{\text {string }}= & -\frac{1}{4 \pi \alpha^{\prime}} \int d^{2} \sigma\left[\sqrt{-h} h^{\alpha \beta} \hat{G}_{\hat{\mu} \hat{\nu}}(X)\right. \\
& \left.+\epsilon^{\alpha \beta} \hat{B}_{\hat{\mu} \hat{\nu}}(X)\right] \partial_{\alpha} \hat{X}^{\hat{\mu}} \partial_{\beta} \hat{X}^{\hat{\nu}} .
\end{aligned}
$$

The worldsheet fields split as $\hat{X}^{\hat{\mu}}=\left(X^{\mu}, Y^{i}\right)$, and it has been made explicit that the spacetime fields do not depend on $Y^{i}$. The $X^{\mu}$ coordinates obey periodic boundary conditions, $X^{\mu}(2 \pi, \tau)=X^{\mu}(0, \tau)$, while the $Y^{i}(\sigma, \tau)$ in principle have winding contributions. However, in light of the discussion in the previous section, we shall restrict our discussion from the beginning to the sector with zero winding, i.e., $Y^{i}(2 \pi, \tau)=Y^{i}(0, \tau)$.

The $(n+d)$-dimensional fields are related to the $n$-dimensional ones by the usual Kaluza-Klein dictionary:

$\hat{G}_{\mu \nu}=g_{\mu \nu}+A_{\mu}^{i} G_{i j} A_{\nu}^{j}, \quad \hat{G}_{\mu i}=G_{i j} A_{\mu}^{j}, \quad \hat{G}_{i j}=G_{i j}$,

$\hat{G}^{\mu \nu}=g^{\mu \nu}, \quad \hat{G}^{\mu i}=-g^{\mu \nu} A_{\nu}^{i}, \quad \hat{G}^{i j}=G^{i j}+A_{\mu}^{i} g^{\mu \nu} A_{\nu}^{j}$,

as well as

$$
\begin{aligned}
\hat{B}_{\mu \nu} & =B_{\mu \nu}-A_{[\mu}^{i} \tilde{A}_{\nu] i}+A_{\mu}^{i} B_{i j} A_{\nu}^{j}, \\
\hat{B}_{\mu i} & =\tilde{A}_{\mu i}-B_{i j} A_{\mu}^{j}, \quad \hat{B}_{i j}=B_{i j} .
\end{aligned}
$$

Using the reduction ansatz (3.6) and (3.7) directly in the Lagrangian action (3.5) leads to a quite unintelligible mess. In the last section we have seen that the appearance of manifest $O(d, d)$ invariance crucially relies on the Hamiltonian formalism. This suggests that the same should happen in the present context. We shall thus rewrite the action (3.5) in Hamiltonian form. In terms of $(n+d)$ dimensional fields this does not require any different computation compared to (2.4) and (2.6), thus giving for the momenta

$$
\hat{P}_{\hat{\mu}}=\frac{1}{2 \pi \alpha^{\prime}}\left[\frac{1}{e} \hat{G}_{\hat{\mu} \hat{\nu}}\left(\dot{\hat{X}}^{\hat{\nu}}-u \hat{X}^{\hat{\nu}}\right)+\hat{B}_{\hat{\mu} \hat{\nu}} \hat{X}^{\hat{\nu}}\right],
$$

with $e$ and $u$ given as in (2.5), and

$$
S_{\text {string }}=\int d^{2} \sigma\left[\hat{P}_{\hat{\mu}} \dot{\hat{X}}^{\hat{\mu}}-e \mathcal{H}-u \mathcal{N}\right] \text {. }
$$

The Virasoro constraints $\mathcal{N}$ and $\mathcal{H}$ are also given by the same expressions as in (2.7), except that all quantities such

\footnotetext{
${ }^{2}$ For the moment we will ignore the coupling to the dilaton, since it is a higher order effect in $\alpha^{\prime}$.
} 
as $G$ and $B$ are replaced by hatted quantities $\hat{G}$ and $\hat{B}$. Splitting the symplectic term and the $\mathcal{N}$ constraint is trivial, since they do not contain spacetime fields:

$$
\begin{aligned}
\hat{P}_{\hat{\mu}} \dot{\hat{X}}^{\hat{\mu}} & =P_{\mu} \dot{X}^{\mu}+P_{i} \dot{Y}^{i}, \\
\mathcal{N} & =P_{\mu} \partial_{\sigma} X^{\mu}+P_{i} \partial_{\sigma} Y^{i}=P_{\mu} \partial_{\sigma} X^{\mu}+\frac{1}{4 \pi \alpha^{\prime}} Z^{M} Z_{M},
\end{aligned}
$$

where we recalled the vector $Z^{M}$ defined in (2.8). The challenge is to express $\mathcal{H}$ in terms of $n$-dimensional fields by using (3.6) and (3.7). After a tedious computation the final result can be expressed in a manifest $O(d, d)$ invariant form:

$$
\begin{aligned}
\mathcal{H}= & \frac{1}{4 \pi \alpha^{\prime}}\left\{g^{\mu \nu} \Pi_{\mu} \Pi_{\nu}-2 g^{\mu \lambda} \mathcal{B}_{\lambda \nu} \Pi_{\mu} \partial_{\sigma} X^{\nu}\right. \\
& +\left(g_{\mu \nu}+g^{\lambda \sigma} \mathcal{B}_{\lambda \mu} \mathcal{B}_{\sigma \nu}\right) \partial_{\sigma} X^{\mu} \partial_{\sigma} X^{\nu} \\
& \left.+\mathcal{H}_{M N}\left(Z^{M}+\mathcal{A}_{\mu}{ }^{M} \partial_{\sigma} X^{\mu}\right)\left(Z^{N}+\mathcal{A}_{\nu}{ }^{N} \partial_{\sigma} X^{\nu}\right)\right\}
\end{aligned}
$$

where

$$
\Pi_{\mu}:=2 \pi \alpha^{\prime} P_{\mu}-\mathcal{A}_{\mu}{ }^{M} Z_{M}, \quad \mathcal{B}_{\mu \nu}:=B_{\mu \nu}+\frac{1}{2} \mathcal{A}_{\mu}{ }^{M} \mathcal{A}_{\nu M} .
$$

The Virasoro constraints $\mathcal{N}$ and $\mathcal{H}$ are clearly $O(d, d)$ invariant, modulo the issue of zero modes discussed in the previous section, that we will revisit in the present context.

There is no reason to keep the noncompact sector in Hamiltonian form. We shall thus eliminate the momenta $P_{\mu}$ by their equations of motion:

$$
\begin{aligned}
2 \pi \alpha^{\prime} P_{\mu} & =e^{-1} g_{\mu \nu} \stackrel{\circ}{ }^{\nu}+\mathcal{B}_{\mu \nu} \partial_{\sigma} X^{\nu}+\mathcal{A}_{\mu}{ }^{M} Z_{M}, \\
\stackrel{\circ}{X}^{\mu} & :=\partial_{\tau} X^{\mu}-u \partial_{\sigma} X^{\mu},
\end{aligned}
$$

and recast the action (3.9) in the mixed form

$$
\begin{aligned}
S_{\text {string }}= & \frac{1}{2 \pi \alpha^{\prime}} \int d^{2} \sigma\left[\frac{1}{2 e} g_{\mu \nu} \stackrel{\circ}{X^{\mu}} \stackrel{\circ}{ }^{\nu}+\left(\mathcal{B}_{\mu \nu} \partial_{\sigma} X^{\nu}+\mathcal{A}_{\mu}{ }^{M} Z_{M}\right) \stackrel{\circ}{X^{\mu}}\right. \\
& -\frac{e}{2} g_{\mu \nu} \partial_{\sigma} X^{\mu} \partial_{\sigma} X^{\nu}+2 \pi \alpha^{\prime} P_{i} \dot{Y}^{i}-\frac{u}{2} Z^{M} Z_{M} \\
& \left.-\frac{e}{2} \mathcal{H}_{M N}\left(Z^{M}+\mathcal{A}_{\mu}{ }^{M} \partial_{\sigma} X^{\mu}\right)\left(Z^{N}+\mathcal{A}_{\nu}{ }^{N} \partial_{\sigma} X^{\nu}\right)\right] .
\end{aligned}
$$

\section{B. Zero mode truncation}

As discussed in the previous section, the formal $O(d, d)$ invariance of the Virasoro constraints $\mathcal{H}$ and $\mathcal{N}$ is broken by the zero mode $p_{i 0}=\frac{1}{2 \pi} \int_{0}^{2 \pi} d \sigma P_{i}$ even in the zero winding sector. Moreover, the symplectic term $p_{i 0} \dot{y}_{0}^{i}$ is another $O(d, d)$ breaking term. Following the discussion in the previous section, we shall thus truncate the action ${ }^{3}$ by projecting out the conjugate pair of zero modes. In order to do this, we set

$$
2 \pi \alpha^{\prime} P_{i}=\partial_{\sigma} \tilde{Y}_{i}
$$

that is an invertible field redefinition for the nonzero modes, and at the same time sets $p_{i 0}=0$ upon taking $\tilde{Y}_{i}(2 \pi, \tau)=\tilde{Y}_{i}(0, \tau)$. By using (3.15) one has $Z^{M}=\partial_{\sigma} Y^{M}$ and the truncated symplectic current can be written in manifestly $O(d, d)$ invariant form:

$$
\int d^{2} \sigma 2 \pi \alpha^{\prime} P_{i} \dot{Y}^{i}=\frac{1}{2} \int d^{2} \sigma \partial_{\sigma} Y^{M} \partial_{\tau} Y_{M}
$$

Before using (3.15) in (3.14), let us discuss spacetime gauge invariances. $n$-dimensional diffeomorphisms are a manifest invariance, upon using

$$
\delta_{\xi} X^{\mu}=-\xi^{\mu}(X), \quad \delta_{\xi} \Phi(X)=\mathcal{L}_{\xi} \Phi(X)+\delta_{\xi} X^{\mu} \partial_{\mu} \Phi(X),
$$

where $\Phi$ generically denotes spacetime fields, and we recalled that, when considering target space fields on the worldsheet, one has to add the extra term in (3.17) to account for the explicit dependence on $X^{\mu}(\sigma, \tau)$. Invariance under two-form gauge transformations $\delta_{\zeta} B_{\mu \nu}=2 \partial_{[\mu} \zeta_{\nu]}$ is also standard. The situation is more subtle for the vector gauge symmetries $\delta_{\lambda} \mathcal{A}_{\mu}{ }^{M}=\partial_{\mu} \lambda^{M}$. The upper component $\delta A_{\mu}^{i}=\partial_{\mu} \lambda^{i}$ is a remnant of $(n+d)$-dimensional diffeomorphisms. This already fixes the transformation for the internal worldsheet coordinates: $\delta_{\lambda} Y^{i}=-\lambda^{i}(X)$. In order to preserve $O(d, d)$, one is led to demand $\delta_{\lambda} \tilde{Y}_{i}=-\tilde{\lambda}_{i}$, so that

$$
\delta_{\lambda} Y^{M}=-\lambda^{M}(X) .
$$

In terms of internal momenta, the lower component gives $\delta_{\lambda} P_{i}=-\frac{1}{2 \pi \alpha^{\prime}} \partial_{\sigma} \tilde{\lambda}_{i}$, that can also be derived by its on-shell expression (3.8) for $\hat{\mu}=i$. This transformation preserves the solution space with $p_{i 0}=0$, since $\delta_{\lambda} p_{i 0}=0$.

Following [6] we introduce the gauge-invariant derivative

$$
D_{\alpha} Y^{M}:=\partial_{\alpha} Y^{M}+\mathcal{A}_{\mu}{ }^{M}(X) \partial_{\alpha} X^{\mu},
$$

which indeed obeys $\delta_{\lambda}\left(D_{\alpha} Y^{M}\right)=0$. The action (3.14), with truncated zero modes according to (3.15), can be finally written as ${ }^{4}$

\footnotetext{
${ }^{3}$ In order to ensure equivalence with the original action, one has to keep track of the $y_{0}^{i}$ equation of motion, which will be done in the following.

${ }^{4}$ Recall that $e$ and $u$ are defined in terms of $h_{\alpha \beta}$ as $e=\sqrt{-h} h_{\sigma \sigma}^{-1}$ and $u=h_{\tau \sigma} h_{\sigma \sigma}^{-1}$.
} 


$$
\begin{aligned}
S= & -\frac{1}{4 \pi \alpha^{\prime}} \int d^{2} \sigma\left[\sqrt{-h} h^{\alpha \beta} g_{\mu \nu} \partial_{\alpha} X^{\mu} \partial_{\beta} X^{\nu}\right. \\
& \left.+\epsilon^{\alpha \beta}\left(B_{\mu \nu} \partial_{\alpha} X^{\mu} \partial_{\beta} X^{\nu}-\mathcal{A}_{\mu}{ }^{M} D_{\alpha} Y_{M} \partial_{\beta} X^{\mu}\right)\right] \\
& +\frac{1}{4 \pi \alpha^{\prime}} \int d^{2} \sigma\left[D_{\sigma} Y^{M} D_{\tau} Y_{M}-u D_{\sigma} Y^{M} D_{\sigma} Y_{M}\right. \\
& \left.-e \mathcal{H}_{M N} D_{\sigma} Y^{M} D_{\sigma} Y^{N}\right] .
\end{aligned}
$$

Not only is $O(d, d)$ manifestly realized, but all terms in the action are gauge invariant under the vector symmetries. For the two terms involving the $B$-field and the bare vector $\mathcal{A}_{\mu}{ }^{M}$ one has to check that

$\delta_{\lambda} \mathcal{A}_{\mu}{ }^{M} \epsilon^{\alpha \beta} D_{\alpha} Y_{M} \partial_{\beta} X^{\mu}=\partial_{\alpha} v^{\alpha}+\frac{1}{2} \lambda_{M} \mathcal{F}_{\mu \nu}{ }^{M} \epsilon^{\alpha \beta} \partial_{\alpha} X^{\mu} \partial_{\beta} X^{\nu}$,

which exactly cancels the nonstandard transformation $\delta_{\lambda} B_{\mu \nu}=\frac{1}{2} \mathcal{F}_{\mu \nu}{ }^{M} \lambda_{M}$ of the $B$-field, thus proving invariance of the action under the spacetime gauge symmetries. The $O(d, d)$ symmetric action (3.20) also has a manifest zero mode local symmetry under

$$
\delta_{\Xi} Y^{M}(\sigma, \tau)=\Xi^{M}(\tau),
$$

that will be used to show equivalence with the (truncated) original sigma model.

Having found the final form (3.20) of the action, let us now show that it provides a consistent truncation of the original theory. To this end we have to determine the zero mode $y_{0}^{i}$ in terms of the untruncated fields so that the original equations of motion are satisfied. The $i$-component of (3.8) gives

$2 \pi \alpha^{\prime} P_{i}=\frac{1}{e} G_{i j}\left(D_{\tau} Y^{j}-u D_{\sigma} Y^{j}\right)+B_{i j} D_{\sigma} Y^{j}-\tilde{A}_{\mu i} \partial_{\sigma} X^{\mu}$

upon using the Kaluza-Klein ansatz (3.6), (3.7) and the definition (3.19) of $D_{\alpha} Y^{M}$. As mentioned above, this also confirms the transformation law $\delta_{\lambda} P_{i}=-\frac{1}{2 \pi \alpha^{\prime}} \partial_{\sigma} \tilde{\lambda}_{i}$ under the vector gauge symmetries. Inverting (3.23) and integrating over $\sigma$ one obtains the original equation for the zero mode $y_{0}^{i}$ :

$$
\dot{y}_{0}^{i}(\tau)=V^{i}(\tau),
$$

with

$V^{M}(\tau)=\frac{1}{2 \pi} \int_{0}^{2 \pi} d \sigma\left[u D_{\sigma} Y^{M}+e \mathcal{H}^{M N} D_{\sigma} Y_{N}-\mathcal{A}_{\mu}{ }^{M} \partial_{\tau} X^{\mu}\right]$,

where (3.15) has been used to ensure $p_{i 0}=0$. Integration of (3.24) then determines $y_{0}^{i}(\tau)$ in terms of the untruncated fields consistent with the equations of motion. One may also verify that (3.24) is invariant under the spacetime gauge symmetry. We note that only the last term above has a nontrivial transformation under $U(1)$, explicitly

$\delta_{\lambda} V^{i}=-\frac{1}{2 \pi} \int_{0}^{2 \pi} d \sigma \partial_{\mu} \lambda^{i} \partial_{\tau} X^{\mu}=-\frac{1}{2 \pi} \int_{0}^{2 \pi} d \sigma \dot{\lambda}^{i}$.

This ensures gauge invariance of (3.24), given that $y_{0}^{i}=$ $\frac{1}{2 \pi} \int_{0}^{2 \pi} d \sigma Y^{i}$ and $\delta_{\lambda} Y^{i}=-\lambda^{i}(X)$. Similarly to the simpler case discussed in the previous section, one could also fix the (arbitrary) function $\tilde{y}_{i 0}$ by supplementing the action (3.20) with the manifestly $O(d, d)$ and spacetime gauge invariant extra equation

$$
\dot{Y}_{0}^{M}=V^{M} .
$$

The extra condition (3.27) can be viewed as a gauge fixing condition for the $\Xi^{M}$ symmetry (3.22). In this respect, the solutions of (3.20) can be embedded into solutions of the original sigma model, up to gauge equivalence.

We end this section by examining the field equations obtained from the action (3.20). The $Y$ field equations are given by a total $\sigma$ derivative:

$$
\partial_{\sigma}\left[D_{\tau} Y^{M}-u D_{\sigma} Y^{M}-e \mathcal{H}^{M N} D_{\sigma} Y_{N}\right]=0,
$$

which makes explicit that the action (3.20) does not determine the dynamics of the zero modes $Y_{0}^{M}(\tau)$. According to (3.28), the quantity in brackets can be an arbitrary function of $\tau$, say $C^{M}(\tau)$, depending on the $\Xi$ gauge. It is easy to see that the $C^{M}(\tau)$ corresponding to the gauge choice (3.27) is $C^{M}=0$, yielding

$$
D_{\tau} Y^{M}-u D_{\sigma} Y^{M}-e \mathcal{H}^{M N} D_{\sigma} Y_{N}=0
$$

that in conformal gauge $(e, u)=(1,0)$ takes the form of a covariantized self-duality relation:

$$
D_{\alpha} Y^{M}=\epsilon_{\alpha}{ }^{\beta} \mathcal{H}^{M N} D_{\beta} Y_{N} .
$$

The first-order equation (3.29) is physically equivalent to the gauge invariant variational equation (3.28). However, it should be kept in mind that it can be used only when discussing pure on-shell properties in a fixed $\Xi^{M}$ gauge, and not otherwise.

The field equations for $X^{\mu}$ resulting from the action (3.20) read

$$
\begin{aligned}
g_{\mu \nu} & \left(\nabla^{2} X^{\nu}+\Gamma_{\lambda \rho}^{\nu} \nabla^{\alpha} X^{\lambda} \nabla_{\alpha} X^{\rho}\right)-\frac{1}{2} \varepsilon^{\alpha \beta}\left[\partial_{\alpha} X^{\nu} \partial_{\beta} X^{\lambda} H_{\mu \nu \lambda}\right. \\
& \left.+2 \partial_{\alpha} X^{\nu} D_{\beta} Y^{M} \mathcal{F}_{\mu \nu M}\right] \\
& +\frac{1}{\sqrt{-h}} \partial_{\sigma} X^{\nu} \mathcal{F}_{\mu \nu}{ }^{M}\left[D_{\tau} Y_{M}-u D_{\sigma} Y_{M}-e \mathcal{H}_{M N} D_{\sigma} Y^{N}\right] \\
& -\frac{e}{2 \sqrt{-h}} \partial_{\mu} \mathcal{H}_{M N} D_{\sigma} Y^{M} D_{\sigma} Y^{N}=0,
\end{aligned}
$$


where $\varepsilon^{\alpha \beta}:=\frac{1}{\sqrt{-h}} \epsilon^{\alpha \beta}$, and $\nabla_{\alpha}$ denote worldsheet covariant derivatives built from $h_{\alpha \beta}$. In conformal gauge, and using the $\Xi$-gauge yielding the first-order equation (3.29), this reduces to a result of Maharana and Schwarz [6]:

$$
\begin{aligned}
g_{\mu \nu} & \left(\square X^{\nu}+\Gamma_{\lambda \rho}^{\nu} \partial^{\alpha} X^{\lambda} \partial_{\alpha} X^{\rho}\right)-\frac{1}{2} \epsilon^{\alpha \beta}\left[\partial_{\alpha} X^{\nu} \partial_{\beta} X^{\lambda} H_{\mu \nu \lambda}\right. \\
& \left.+2 \partial_{\alpha} X^{\nu} D_{\beta} Y^{M} \mathcal{F}_{\mu \nu M}\right] \\
& -\frac{1}{4} \partial_{\mu} \mathcal{H}_{M N} D_{\alpha} Y^{M} D^{\alpha} Y^{N}=0
\end{aligned}
$$

where we used again the first-order duality relation (3.30) to recast the last term in a manifestly Lorentz invariant form.

Finally, the equations of motion of the worldsheet metric, obtained by varying with respect to $e$ and $u$, are given by

$$
\begin{aligned}
-\frac{\delta S}{\delta e}= & \mathcal{H}=\frac{1}{4 \pi \alpha^{\prime}}\left[e^{-2} g_{\mu \nu} \stackrel{\circ}{ }^{\mu} \stackrel{\circ}{ }^{\nu}+g_{\mu \nu} \partial_{\sigma} X^{\mu} \partial_{\sigma} X^{\nu}\right. \\
& \left.+\mathcal{H}_{M N} D_{\sigma} Y^{M} D_{\sigma} Y^{N}\right], \\
-\frac{\delta S}{\delta u}= & \mathcal{N}=\frac{1}{4 \pi \alpha^{\prime}}\left[2 e^{-1} g_{\mu \nu} \stackrel{\circ}{ }^{\mu} \partial_{\sigma} X^{\nu}+D_{\sigma} Y^{M} D_{\sigma} Y_{M}\right],
\end{aligned}
$$

where $\stackrel{\circ}{X}^{\mu}=\partial_{\tau} X^{\mu}-u \partial_{\sigma} X^{\mu}$.

In the whole discussion so far we glossed over the fate of worldsheet diffeomorphisms. In an Appendix we examine this issue in great detail and provide the explicit realization of diffeomorphism symmetry in the action (3.20). In particular, we prove that the diffeomorphism transformations,

$$
\begin{aligned}
\delta_{\xi} X^{\mu} & =\xi^{\alpha} \partial_{\alpha} X^{\mu}, \\
\delta_{\xi} Y^{M} & =\xi^{\alpha} \partial_{\alpha} Y^{M}-\xi^{\tau}\left[D_{\tau} Y^{M}-u D_{\sigma} Y^{M}-e \mathcal{H}^{M N} D_{\sigma} Y_{N}\right],
\end{aligned}
$$

are an off-shell invariance of the action.

\section{ANOMALIES}

In the previous sections we have constructed the manifestly $O(d, d)$ invariant worldsheet sigma model (3.20). Worldsheet diffeomorphism invariance is not manifest, but it is extensively discussed in Appendix A, as is the cancellation of gravitational anomalies.

The sigma model (3.20) seems a good starting point to perform worldsheet perturbation theory in an $O(d, d)$ covariant way, to all orders in $\alpha^{\prime}$. However, from the analysis of the low-energy spacetime theory, it has been recently found $[19,20]$ that the $B$-field acquires a nontrivial transformation under $O(d, d)$ at first order in $\alpha^{\prime}$. This is reminiscent of the original Green-Schwarz mechanism [34] in type I or heterotic string theory. Similarly to the heterotic worldsheet theory that contains chiral fermions in both the gravitational and gauge sectors, the $O(d, d)$ sigma model (3.20) is defined in terms of chiral bosons à la FloreaniniJackiw [35]. This suggests that the novel $O(d, d)$ GreenSchwarz mechanism found in [19] can also be explained in terms of worldsheet anomalies, as we will show here.

\section{A. Framelike worldsheet action}

In this section we will focus on the $Y$ sector of the sigma model. The aim is to exhibit two-dimensional anomalies that underlie the $O(d, d)$ Green-Schwarz deformation. Since the $\alpha^{\prime}$ deformation of [19] does not involve the Kaluza-Klein gauge fields $\mathcal{A}_{\mu}{ }^{M}$, we will set them to zero and focus on the action

$S_{Y}=\frac{1}{4 \pi \alpha^{\prime}} \int d^{2} \sigma\left[\partial_{\sigma} Y^{M} \partial_{\tau} Y_{M}-\mathcal{H}_{M N}(X) \partial_{\sigma} Y^{M} \partial_{\sigma} Y^{N}\right]$.

It is convenient to rewrite this action in terms of a frame formalism, which we briefly introduce now. The generalized metric can be written in terms of frame fields as $[7,36,37]$

$$
\mathcal{H}_{M N}(x)=E_{M}{ }^{A}(x) h_{A B} E_{N}^{B}(x),
$$

where we have introduced the frame field $E_{M}{ }^{A}$, and a $S O(d) \times S O(d)$ constant metric $h_{A B}$. The $O(d, d)$ invariant metric $\eta_{M N}$, on the other hand, can be written as

$$
\eta_{M N}=E_{M}{ }^{A}(x) \eta_{A B} E_{N}{ }^{B}(x),
$$

where $\eta_{A B}$ has the same numerical form as $\eta_{M N}$. This choice implies that the frame field itself is an $O(d, d)$ matrix. In the following we will use $\eta^{A B}$ and $\eta_{A B}$ to raise and lower flat indices. Denoting the inverse vielbein by $E_{A}{ }^{M}$, such that $E_{M}{ }^{A} E_{A}{ }^{N}=\delta_{M}{ }^{N}$ and $E_{A}{ }^{M} E_{M}{ }^{B}=\delta_{A}{ }^{B}$, the raising and lowering of indices is then consistent with taking inverses:

$$
E_{A}{ }^{M}=\eta_{A B} \eta^{M N} E_{N}{ }^{B} .
$$

Furthermore, $h_{A B}$ satisfies the constraints $h_{A C} \eta^{C D} h_{C B}=$ $\eta_{A B}$ and $\eta^{A B} h_{A B}=0$. In this formalism one has in addition to rigid $O(d, d)$ transformations local $S O(d) \times S O(d)$ transformations:

$$
\begin{aligned}
& \delta_{\lambda} E_{M}{ }^{A}(x)=-\lambda_{B}{ }^{A}(x) E_{M}{ }^{B}(x), \\
& \delta_{\lambda} E_{A}{ }^{M}(x)=\lambda_{A}{ }^{B}(x) E_{B}{ }^{M}(x),
\end{aligned}
$$

where the parameters $\lambda_{A}{ }^{B}$ obey the $S O(d) \times S O(d)$ condition $\lambda_{(A}{ }^{C} h_{B) C}=0$ and the $O(d, d)$ condition $\lambda_{(A}{ }^{C} \eta_{B) C}=\lambda_{(A B)}=0$. The $S O(d) \times S O(d)$ preserving condition on $\lambda$ can be conveniently rewritten as 
$\lambda_{A C} h_{B}^{C}+\lambda_{B C} h_{A}^{C}=\lambda_{A C} h_{B}^{C}-h^{C}{ }_{A} \lambda_{C B}=[\lambda, h]_{A B}=0$,

where we used $h=h^{\mathrm{T}}$ as a matrix.

In the following it will be important to separate irreducible $S O(d) \times S O(d)$ representations from any tensor with indices $A, B=1, \ldots, 2 d$. This can be achieved by use of projection operators

$$
\Pi_{ \pm B}^{A}=\frac{1}{2}\left(\delta_{B}^{A} \pm h_{B}^{A}\right)
$$

which, thanks to the constraints stated after (4.4), are orthogonal and obey $\Pi_{ \pm}^{2}=\Pi_{ \pm}$, the completeness relation $\mathbf{1}=\Pi_{+}+\Pi_{-}$and $\operatorname{Tr} \Pi_{ \pm}^{ \pm}=d$. This allows us to decompose an arbitrary vector $V^{A}$ as

$$
V^{A}=V_{+}^{A}+V_{-}^{A}=V^{\underline{A}}+V^{\bar{A}}
$$

where we shall denote by $\underline{A}$ an index projected via $\Pi_{+}$and $\bar{A}$ an index projected by $\Pi_{-}$. This way $\underline{A}$ and $\bar{A}$ indices carry the $(d, 0)$ and $(0, d)$ representations of $S O(d) \times S O(d)$, respectively. Higher tensors decompose analogously. For instance, the gauge parameter $\lambda_{A B}$ decomposes as

$$
\lambda_{A B}=\lambda_{\underline{A B}}+\lambda_{\overline{A B}},
$$

with $\lambda_{(\underline{A B})}=0, \lambda_{(\overline{A B})}=0$, where the vanishing of the off-diagonal components $\lambda_{\underline{A} \bar{B}}$ and $\lambda_{\bar{A}} \underline{B}$ follows since $\left[\lambda, \Pi_{ \pm}\right]=0$ by (4.6). This fact makes it manifest that the gauge group is only $S O(d) \times S O(d)$.

Let us finally define the (composite) gauge fields for the $S O(d) \times S O(d)$ gauge symmetry. We start from the Maurer-Cartan form

$$
W_{\mu A B}:=E_{A}{ }^{M} \partial_{\mu} E_{M B}=-W_{\mu B A},
$$

that can be decomposed into connections $Q_{\mu A B}$ of $S O(d) \times S O(d):$

$Q_{\mu A B}:=\left(\Pi_{+} W_{\mu} \Pi_{+}\right)_{A B}+\left(\Pi_{-} W_{\mu} \Pi_{-}\right)_{A B}=Q_{\mu \underline{A B}}+Q_{\mu \overline{A B}}$,

satisfying $Q_{\mu(\underline{A B})}=0, Q_{\mu(\overline{A B})}=0$, and a tensor $P_{\mu A B}$ in the $(d, d)$ representation:

$$
P_{\mu A B}:=\left(\Pi_{+} W_{\mu} \Pi_{-}\right)_{A B}+\left(\Pi_{-} W_{\mu} \Pi_{+}\right)_{A B}=P_{\mu \underline{A} \bar{B}}+P_{\mu \bar{A} \underline{B}},
$$

where $P_{\mu \bar{A} \underline{B}}=-P_{\mu \underline{B} \bar{A}}$. More precisely, the transformation properties under (4.5) are

$$
\begin{aligned}
& \delta_{\lambda} Q_{\mu \underline{A B}}=-\mathcal{D}_{\mu} \lambda_{\underline{A B}}:=-\left(\partial_{\mu} \lambda_{\underline{A B}}+\left[Q_{\mu}, \lambda\right]_{\underline{A B}}\right), \\
& \delta_{\lambda} Q_{\mu \overline{A B}}=-\mathcal{D}_{\mu} \lambda_{\overline{A B}}:=-\left(\partial_{\mu} \lambda_{\overline{A B}}+\left[Q_{\mu}, \lambda\right]_{\overline{A B}}\right), \\
& \delta_{\lambda} P_{\mu \underline{A} \bar{B}}=\lambda_{\underline{A}} \underline{C} P_{\mu \underline{C} \bar{B}}+\lambda_{\bar{B}} \bar{c} P_{\mu \underline{A} \bar{C}},
\end{aligned}
$$

or, without splitting, $\delta_{\lambda} Q_{\mu A B}=-\mathcal{D}_{\mu} \lambda_{A B}$ and $\delta_{\lambda} P_{\mu A B}=$ $\left[\lambda, P_{\mu}\right]_{A B}$. Finally, the Maurer-Cartan form obeys the zero curvature identity $d W+W^{2}=0$, which gives rise to the Bianchi identities

$$
\begin{aligned}
R_{\mu \nu} & \equiv \partial_{\mu} Q_{\nu}-\partial_{\nu} Q_{\mu}+\left[Q_{\mu}, Q_{\nu}\right]=-\left[P_{\mu}, P_{\nu}\right], \\
\mathcal{D}_{[\mu} P_{\nu]} & \equiv \partial_{[\mu} P_{\nu]}+\left[Q_{[\mu}, P_{\nu]}\right]=0,
\end{aligned}
$$

where we used matrix notation.

After this review of the frame formalism we now return to the worldsheet theory (4.1). We perform the field redefinition that flattens the worldsheet fields $Y^{M}$ :

$$
Y^{M}=E_{A}{ }^{M}(X) Y^{A} .
$$

The worldsheet derivatives $\partial_{\alpha} Y^{M}$ then become

$$
\partial_{\alpha} Y^{M}=E_{A}{ }^{M}\left(\partial_{\alpha} Y^{A}+W_{\alpha}{ }_{B}{ }_{B} Y^{B}\right)=E_{A}{ }^{M} \hat{\mathcal{D}}_{\alpha} Y^{A},
$$

where we introduced the pullback $W_{\alpha A B}:=\partial_{\alpha} X^{\mu} W_{\mu A B}$ and the hatted covariant derivative

$\hat{\mathcal{D}}_{\alpha} Y^{A}:=\partial_{\alpha} Y^{A}+W_{\alpha}{ }_{B} Y^{B}=\mathcal{D}_{\alpha} Y^{A}+P_{\alpha}{ }_{B}^{A} Y^{B}$,

that differs from the $S O(d) \times S O(d)$ covariant derivative $\mathcal{D}_{\alpha}$, which is defined by this equation, by the above coupling to $P_{\alpha A B}$. The action (4.1) can thus be written as

$S_{Y}=\frac{1}{4 \pi \alpha^{\prime}} \int d^{2} \sigma\left[\hat{\mathcal{D}}_{\sigma} Y^{A} \hat{\mathcal{D}}_{\tau} Y_{A}-h_{A B} \hat{\mathcal{D}}_{\sigma} Y^{A} \hat{\mathcal{D}}_{\sigma} Y^{B}\right]$.

The zero-mode symmetry $\delta_{\Xi} Y^{M}=\Xi^{M}$, with $\partial_{\sigma} \Xi^{M}=0$, now turns into

$$
\delta_{\Xi} Y^{A}=\Xi^{A}, \quad \text { where } \hat{\mathcal{D}}_{\sigma} \Xi^{A}=0 .
$$

By means of the projectors $\Pi_{ \pm}$one can split $Y^{A}$ into $S O(d) \times S O(d)$ representations: $Y^{A}=Y^{A}+Y^{\bar{A}}$ under which the action decomposes as

$$
\begin{aligned}
S_{Y}= & \frac{1}{2 \pi \alpha^{\prime}} \int d^{2} \sigma\left[\hat{\mathcal{D}}_{\sigma} Y^{\underline{A}} \hat{\mathcal{D}}_{-} Y_{\underline{A}}+\hat{\mathcal{D}}_{\sigma} Y^{\bar{A}} \hat{\mathcal{D}}_{+} Y_{\bar{A}}\right] \\
= & \frac{1}{2 \pi \alpha^{\prime}} \int d^{2} \sigma\left[\left(\mathcal{D}_{\sigma} Y^{\underline{A}}+P_{\sigma^{\underline{A}}} Y_{\bar{B}} Y^{\bar{B}}\right)\left(\mathcal{D}_{-} Y_{\underline{A}}+P_{-\underline{A} \bar{C}} Y^{\bar{C}}\right)\right. \\
& \left.\quad+\left(\mathcal{D}_{\sigma} Y^{\bar{A}}+P_{\sigma}{ }_{\underline{B}}^{\bar{A}} Y^{\underline{B}}\right)\left(\mathcal{D}_{+} Y_{\bar{A}}+P_{+\bar{A}} \underline{C} Y^{\underline{C}}\right)\right], \quad(4.20
\end{aligned}
$$

with $\mathcal{D}_{ \pm}=\frac{1}{2}\left(\mathcal{D}_{\tau} \pm \mathcal{D}_{\sigma}\right)$. Under a local $S O(d) \times S O(d)$ transformation, 


$$
\delta_{\lambda} Y^{A}=\lambda^{A}{ }_{B}(X) Y^{B},
$$

the hatted derivatives $\hat{\mathcal{D}}_{\alpha} Y^{A}$ transform covariantly, i.e.,

$$
\delta_{\lambda}\left(\hat{\mathcal{D}}_{\alpha} Y^{A}\right)=\lambda^{A}{ }_{B} \hat{\mathcal{D}}_{\alpha} Y^{B} .
$$

It is thus clear that the action (4.18) is invariant under (spacetime) local $S O(d) \times S O(d)$, provided one transforms simultaneously $Y^{A}$ and $W_{\alpha}^{A B}$.

We now turn to a general discussion of potential anomalies in this model, which will be computed explicitly in the next subsection. It must first be emphasized that the above invariance under $S O(d) \times S O(d)$ is not a genuine symmetry of the worldsheet theory, since the background fields (target space fields) need to be transformed as well. Nonetheless, it is an important consistency condition that any two configurations of target space fields that are gauge equivalent (from the target space point of view) give rise to equivalent worldsheet theories. It is this property that may become anomalous. This is precisely analogous to heterotic string theory where the coupling to target space Yang-Mills gauge fields is quantum-mechanically inconsistent unless the Green-Schwarz mechanism is invoked [29].

While the $S O(d) \times S O(d)$ is not a genuine symmetry of the worldsheet theory, one can derive consequences from this invariance property:

$$
\int d^{2} \sigma\left[\frac{\delta S}{\delta W_{\alpha}^{A B}} \hat{\mathcal{D}}_{\alpha} \lambda^{A B}\right]=-\frac{1}{4 \pi \alpha^{\prime}} \int d^{2} \sigma\left[\lambda^{A B} \hat{\mathcal{D}}_{\alpha} \mathcal{J}_{A B}^{\alpha}\right]=0,
$$

where we assumed that the $Y^{\prime}$ s are on-shell: $\frac{\delta S}{\delta Y^{A}}=0$, and we have defined

$$
\mathcal{J}_{A B}^{\alpha}:=4 \pi \alpha^{\prime} \frac{\delta S}{\delta W_{\alpha}^{A B}},
$$

which reads in components

$\mathcal{J}_{A B}^{\tau}=-Y_{[A} \hat{\mathcal{D}}_{\sigma} Y_{B]}, \quad \mathcal{J}_{A B}^{\sigma}=-Y_{[A}\left(\hat{\mathcal{D}}_{\tau} Y_{B]}-2 h_{B] C} \hat{\mathcal{D}}_{\sigma} Y^{C}\right)$.

While the $\mathcal{J}_{A B}^{\alpha}$ are not conserved $S O(d) \times S O(d)$ currents the relation (4.23) implies the projected "conservation" law

$\Pi_{+}^{A C} \Pi_{+}^{B D}\left(\hat{\mathcal{D}}_{\alpha} \mathcal{J}^{\alpha}\right)_{C D}=0, \quad \Pi_{-}^{A C} \Pi_{-}^{B D}\left(\hat{\mathcal{D}}_{\alpha} \mathcal{J}^{\alpha}\right)_{C D}=0$,

or, using manifest $S O(d) \times S O(d)$ indices,

$$
\begin{aligned}
& \mathcal{D}_{\alpha} \mathcal{J}_{\underline{A} \underline{B}}^{\alpha}+P_{\alpha \underline{A}}^{\bar{C}} \mathcal{J}_{\bar{C} \underline{B}}^{\alpha}-P_{\alpha \underline{B}}{ }^{\bar{C}} \mathcal{J}_{\bar{C} \underline{A}}^{\alpha}=0, \\
& \mathcal{D}_{\alpha} \mathcal{J}_{\bar{A} \bar{B}}^{\alpha}+P_{\alpha \bar{A}} \underline{\underline{C}} \mathcal{J}_{\underline{C} \bar{B}}^{\alpha}-P_{\alpha \bar{B}} \underline{\mathcal{J}}_{\underline{C} \bar{A}}^{\alpha}=0 .
\end{aligned}
$$

One can explicitly verify that (4.27) holds upon using the equation of motion

$$
\hat{\mathcal{D}}_{\sigma}\left(\hat{\mathcal{D}}_{\tau} Y^{A}-h^{A}{ }_{B} \hat{\mathcal{D}}_{\sigma} Y^{B}\right)=0 .
$$

Despite (4.27) not being a standard conservation law, the above result shows that the free theory (where $W_{\mu}^{A B}$ is set to zero) does have conserved $S O(d) \times S O(d)$ currents $j_{\underline{A B}}^{\alpha}$ and $j_{\overline{A B}}^{\alpha}$ :

$$
\begin{array}{ll}
j_{\underline{A B}}^{\tau}=-Y_{[\underline{A}} \partial_{\sigma} Y_{\underline{B}]}, & j_{\underline{A B}}^{\sigma}=-Y_{[\underline{A}}\left(\partial_{\tau}-2 \partial_{\sigma}\right) Y_{\underline{B}]}, \\
j_{\overline{A B}}^{\tau}=-Y_{[\bar{A}} \partial_{\sigma} Y_{\bar{B}]}, & j_{\overline{A B}}^{\sigma}=-Y_{[\bar{A}}\left(\partial_{\tau}+2 \partial_{\sigma}\right) Y_{\bar{B}]},
\end{array}
$$

obeying the usual conservation law $\partial_{\alpha} j_{\underline{A B}}^{\alpha}=0, \partial_{\alpha} j_{\frac{A B}{A B}}^{\alpha}=0$. This emergence of conserved currents can be understood most clearly in the original form of the action (4.1). In the free limit $\mathcal{H}_{M N}$ reduces to a constant (its background value), which is invariant under a global $S O(d) \times$ $S O(d)$, hence giving rise to conserved Noether currents, given by (4.29).

In order to employ the above action for a perturbative quantum field theory treatment let us inspect the linearized coupling to $W_{\mu}^{A B}$

$$
\begin{aligned}
S_{Y}= & \frac{1}{2 \pi \alpha^{\prime}} \int d^{2} \sigma\left[\partial_{\sigma} Y^{A} \partial_{-} Y_{\underline{A}}+\partial_{\sigma} Y^{\bar{A}} \partial_{+} Y_{\bar{A}}+\frac{1}{2} W_{\alpha}^{A B} J_{A B}^{\alpha}\right] \\
& +\mathcal{O}\left(W^{2}\right) \\
= & \frac{1}{2 \pi \alpha^{\prime}} \int d^{2} \sigma\left[\partial_{\sigma} Y^{A} \partial_{-} Y_{\underline{A}}+\partial_{\sigma} Y^{\bar{A}} \partial_{+} Y_{\bar{A}}+\frac{1}{2} Q_{\alpha}^{\underline{A B}} j_{\underline{A B}}^{\alpha}\right. \\
& \left.+\frac{1}{2} Q_{\alpha}^{\overline{A B}} j_{\overline{A B}}^{\alpha}+\frac{1}{2} P^{\bar{A} \overline{\bar{B}}} t_{\underline{A} \bar{B}}^{\alpha}\right]+\mathcal{O}\left(W^{2}\right),
\end{aligned}
$$

which involves the usual interaction term of gauge field and current, but also a coupling to $P_{\mu}^{A B}$, through the $(d, d)$ tensor $t_{\underline{A} \bar{B}}^{\alpha}$ defined as

$$
\begin{aligned}
& t_{\underline{A} \bar{B}}^{\tau}=Y^{\bar{B}} \partial_{\sigma} Y^{A}-Y^{A} \partial_{\sigma} Y^{\bar{B}}, \\
& t_{\underline{A} \bar{B}}^{\sigma}=Y^{\bar{B}}\left(\partial_{\tau}-2 \partial_{\sigma}\right) Y^{\underline{A}}-Y^{A}\left(\partial_{\tau}+2 \partial_{\sigma}\right) Y^{\bar{B}} .
\end{aligned}
$$

In a perturbative treatment of the above action, one splits the "external" coordinate fields $X^{\mu}(\tau, \sigma)$ into a background $X_{0}^{\mu}$ plus fluctuations $\pi^{\mu}$ while, for the present purpose, the $Y^{A}$ can be treated as purely quantum. Among others, the action (4.30) produces the worldsheet vertices $W_{\mu}^{A B}\left(X_{0}\right) \partial_{\alpha} X_{0}^{\mu} J_{A B}^{\alpha}(Y)$ and $\partial_{\mu} W_{\nu}^{A B}\left(X_{0}\right) \pi^{\mu} \partial_{\alpha} \pi^{\nu} J_{A B}^{\alpha}(Y)$. In principle, these two can combine with the vertex $H_{\mu \nu \lambda}\left(X_{0}\right) \epsilon^{\alpha \beta} \partial_{\alpha} X_{0}^{\mu} \partial_{\beta} \pi^{\nu} \pi^{\lambda}$, arising from the expansion of $\int_{\Sigma} B$ in (3.20), to produce a two-loop contribution of the schematic form

$$
\alpha^{\prime} \partial_{\alpha} X_{0}^{\mu} \partial^{\alpha} X_{0}^{\nu}\left[H_{\mu}{ }^{\lambda \sigma} \operatorname{tr}(W \wedge d W)_{\nu \lambda \sigma}\right] .
$$

Its divergent part contributes to the $\beta$-functional of the metric $g_{\mu \nu}$, thus modifying the Einstein equation 
$R_{\mu \nu}=\frac{1}{4} H_{\mu}^{\lambda \sigma} H_{\nu \lambda \sigma}+\cdots$ by the above $\alpha^{\prime}$ correction. This is analogous to the correction underpinning the original Green-Schwarz deformation [38] that results in the redefinition $H \rightarrow H+\frac{\alpha^{\prime}}{4 \pi}\left(\omega_{3}(A)-\omega_{3}(\omega)\right)$. In the present case, the structure (4.32) matches with the $\alpha^{\prime}$ deformation found in [19].

Let us now discuss the appearance of potential anomalies in a little more detail. Focusing on the $Q$-dependent part of (4.32), the very existence and the precise structure of the correction is determined by the one-loop two-point functions

$$
\left\langle j_{\alpha} \frac{A}{\alpha} j_{\beta}^{\frac{C}{B}} \underline{D}\right\rangle, \quad\left\langle j_{\alpha}^{\bar{A}} \bar{B} j_{\beta}^{\bar{C}} \bar{D}\right\rangle .
$$

In the standard heterotic string context, the Green-Schwarz deformation is driven by the worldsheet chiral anomaly: the components $J_{+}^{a b}$ of the gauge current and $J_{-}^{\mu \nu}$ of the Lorentz current vanish identically in the classical theory, due to the chiral nature of the fermions $\lambda^{a}$ and $\psi^{\mu}$. The chiral anomaly, however, gives rise to nonvanishing two-point functions $\left\langle J_{+} J_{-}\right\rangle$at one-loop that are ultimately responsible for the Green-Schwarz deformation.

The situation for the model (4.30) is not exactly the same, but quite similar. Indeed, none of the currents (4.29) vanish identically, but half of them are classically trivial: when written in light-cone coordinates, one has

$$
\begin{aligned}
& j_{+}^{\dot{A}_{+} \underline{B}}=Y^{[\underline{A}}\left(\partial_{+}-2 \partial_{-}\right) Y^{\underline{B}]}, \quad j^{\stackrel{A}{=} \underline{B}}=Y^{[\underline{A}} \partial_{-} Y^{\underline{B}]}, \\
& j_{+}^{\bar{A} \bar{B}}=-Y^{[\bar{A}} \partial_{+} Y^{\bar{B}]}, \quad j_{-}^{\bar{A} \bar{B}}=Y^{[\bar{A}}\left(2 \partial_{+}-\partial_{-}\right) Y^{\bar{B}]} .
\end{aligned}
$$

Let us recall that the free-field equations $\partial_{\sigma} \partial_{-} Y^{A}=0$, $\partial_{\sigma} \partial_{+} Y^{\bar{A}}=0$ imply the chirality conditions $\partial_{-} Y^{A}=0$ and $\partial_{+} Y^{\bar{A}}=0$ except for the zero-modes $Y_{0}^{A}(\tau)$. These, however, are pure gauge, thanks to the free-field symmetry $\delta_{\Xi} Y^{A}=\Xi^{A}(\tau)$, and can be fixed to zero. This shows that, upon gauge fixing, the classical currents obey

$$
\begin{array}{ll}
j_{-}^{\underline{A} \underline{B}}=0, & \partial_{-} j_{+}^{\underline{A} \underline{B}}=0, \\
j_{+}^{\bar{A} \bar{B}}=0, & \partial_{+} j_{-}^{\bar{A} \bar{B}}=0 .
\end{array}
$$

Focusing on the left-moving sector, at the quantum level the two-point function $\left\langle j_{+} \underline{A} \underline{\underline{B}} j_{+}^{\underline{C}} \underline{D}\right\rangle$ is certainly nonvanishing, which implies that the classical relations (4.35) cannot hold. Indeed, the naive Ward identities read

$$
\begin{aligned}
& p_{-}\left\langle j_{+}^{\underline{A}} \underline{\underline{B}}(p) j_{+}^{\underline{C}} \underline{D}(-p)\right\rangle+p_{+}\left\langle j_{-}^{\underline{A} \underline{B}}(p) j_{+}^{\underline{C}} \underline{\underline{D}}(-p)\right\rangle=0, \\
& p_{+}\left\langle j_{-}^{\underline{A} \underline{B}}(p) j_{-}^{\underline{C} \underline{D}}(-p)\right\rangle+p_{-}\left\langle j_{+}^{\underline{A}} \underline{\underline{B}}(p) j_{-}^{\underline{C}} \underline{\underline{D}}(-p)\right\rangle=0,
\end{aligned}
$$

so that, if $\left\langle j_{-} j_{+}\right\rangle=0$ continues to hold, they cannot be satisfied, leading to an anomaly. In the following we will provide a scheme that ensures $j_{-}=0$ in all two-point functions, so that the above shows that $\partial_{-} j_{+}=0$ cannot be satisfied if $\left\langle j_{+} j_{+}\right\rangle$is nonzero.

\section{B. Anomalies of Floreanini-Jackiw}

We will now confirm the existence of an anomaly by computing the above two-point functions. It is sufficient to focus on the free part of the action, which consists of $d$ leftmoving and $d$ right-moving so-called Floreanini-Jackiw bosons. Focusing first on the left-moving sector we consider the action

$$
S=\frac{1}{2 \pi \alpha^{\prime}} \int d^{2} x \partial_{-} \phi^{a} \partial_{\sigma} \phi_{a},
$$

where $a=1, \ldots, d$. Here we have changed notation to emphasize that the following holds generally for the Floreanini-Jackiw model. The action is invariant under rigid $S O(d)$ transformations given by

$$
\delta \phi^{a}=\lambda^{a b} \phi_{b}
$$

where indices are lowered and raised with the $S O(d)$ metric $\delta_{a b}$ and its inverse, respectively. The Noether currents associated with $S O(d)$ are given by

$j_{-}^{a b}=\frac{1}{\alpha^{\prime}} \phi^{[a} \partial_{-} \phi^{b]}, \quad j_{+}^{a b}=\frac{1}{\alpha^{\prime}} \phi^{[a}\left(\partial_{+}-2 \partial_{-}\right) \phi^{b]}$,

and are conserved, obeying $\partial_{+} j_{-}^{a b}+\partial_{-} j_{+}^{a b}=0$, thanks to the field equation

$$
\partial_{\sigma} \partial_{-} \phi^{a}=\left(\partial_{+}-\partial_{-}\right) \partial_{-} \phi^{a}=0 .
$$

Here and in what follows we shall denote the light-cone coordinates by $x^{ \pm}=\tau \pm \sigma$, so that $\partial_{ \pm}=\frac{1}{2}\left(\partial_{\tau} \pm \partial_{\sigma}\right)$.

The action and the field equations are Lorentz invariant, with $\phi^{a}$ transforming in a nonstandard way ${ }^{5}$ :

$$
\delta_{L} \phi^{a}=\omega x^{+}\left(\partial_{+}-\partial_{-}\right) \phi^{a}=\omega x^{+} \partial_{\sigma} \phi^{a},
$$

in contrast with the scalar transformation

$$
\delta_{L} \varphi=\omega\left(x^{+} \partial_{+}-x^{-} \partial_{-}\right) \varphi .
$$

Under a Lorentz transformation, a standard one-form $A_{\alpha}$ transforms as

$$
\delta_{L} A_{ \pm}=\omega\left(x^{+} \partial_{+}-x^{-} \partial_{-}\right) A_{ \pm} \pm \omega A_{ \pm},
$$

\footnotetext{
${ }^{5}$ The action actually has the much larger symmetry $\delta \phi^{a}=\xi^{1}\left(x^{+}\right) \partial_{1} \phi^{a}+\xi^{-}(\tau) \partial_{-} \phi^{a}$, where $\xi^{1}$ and $\xi^{-}$are arbitrary functions of their arguments. This is a manifestation of the infinite-dimensional conformal symmetry in two dimensions. Note that the "second Lorentz symmetry" with $\xi^{-}(\tau)=\lambda \tau$ is trivial in the "chiral gauge" $\partial_{-} \phi^{a}=0$ that we shall employ and hence this symmetry will not play any role in what follows.
} 
while the above current transforms according to

$$
\begin{aligned}
& \delta_{L} j_{-}^{a b}=\omega x^{+}\left(\partial_{+}-\partial_{-}\right) j_{-}^{a b}, \\
& \delta_{L} j_{+}^{a b}=\omega x^{+}\left(\partial_{+}-\partial_{-}\right) j_{+}^{a b}+\omega j_{+}^{a b}+\omega j_{-}^{a b} .
\end{aligned}
$$

To be more precise, the above Lorentz invariance is present if the theory is defined on the plane, i.e., on twodimensional Minkowski space, but we should recall that for the string we defined the theory on the cylinder, where $\tau \in \mathbb{R}$ and $\sigma \in[0,2 \pi]$. The fields can then be expanded in Fourier modes:

$$
\phi^{a}(\tau, \sigma)=\sum_{n \in \mathbb{Z}} \phi_{n}^{a}(\tau) e^{i n \sigma}, \quad\left(\phi_{n}^{a}\right)^{*}=\phi_{-n}^{a} .
$$

Since the Lorentz transformations (4.41) depend explicitly on $\sigma$ they do not respect the periodicity conditions, and so there are no well-defined Lorentz transformations for the Fourier modes. Thus, on the cylinder Lorentz invariance is lost. It is also important to note that the action (4.37) does not contain the zero-mode $\phi_{0}^{a}(\tau)$. This is reflected in the $\tau$-local symmetry

$$
\delta_{\xi} \phi^{a}(\tau, \sigma)=\xi^{a}(\tau)
$$

that shifts $\phi_{0}^{a}$ by an arbitrary function, while leaving the nonzero modes $\phi_{n}^{a}$ inert.

Upon gauge fixing $\phi_{0}^{a}(\tau)=0$, the field equation is equivalent to the chirality condition $\partial_{-} \phi^{a}=0$, showing that one can consider the on-shell equivalent current

$$
j_{-}^{a b}=0, \quad j_{+}^{a b}=\frac{1}{\alpha^{\prime}} \phi^{[a} \partial_{+} \phi^{b]} .
$$

Notice that the above current $j_{\alpha}^{a b}$ transforms as a chiral oneform, i.e.,

$$
\delta_{L} j_{-}^{a b}=0, \quad \delta_{L} j_{+}^{a b}=\omega x^{+} \partial_{+} j_{+}^{a b}+\omega j_{+}^{a b},
$$

only on-shell, upon using $\partial_{-} \phi^{a}=0$.

By using the mode expansion (4.45) one can rewrite the free action as

$$
S=\frac{1}{2 \alpha^{\prime}} \int d \tau \sum_{n \neq 0}\left[i n \phi_{a n} \dot{\phi}_{-n}^{a}-n^{2} \phi_{a n} \phi_{-n}^{a}\right] .
$$

Upon decomposing the $\phi_{n}^{a}$ into two sets (i.e., upon picking a polarization) this action can be brought immediately into Hamiltonian form, with the first term taking the standard $p \dot{q}$ form. It is then straightforward to perform canonical quantization, leading to the equal-time commutation relations

$$
\left[\phi_{n}^{a}, \phi_{m}^{b}\right]=\frac{\alpha^{\prime}}{m} \delta^{a b} \delta_{n+m}, \quad n, m \neq 0,
$$

with the usual notation $\delta_{n+m}:=\delta_{n+m, 0}$. We assume from now on that the zero-mode has been gauge fixed to zero. Ordinary creation-annihilation operators are defined as

$A_{n}^{a}:=\sqrt{\frac{n}{\alpha^{\prime}}} \phi_{-n}^{a}, \quad A_{n}^{\dagger a}:=\sqrt{\frac{n}{\alpha^{\prime}}} \phi_{n}^{a}, \quad n>0$,

and obey

$$
\left[A_{n}^{a}, A_{m}^{\dagger b}\right]=\delta^{a b} \delta_{n m}
$$

The mode expansion of the quantum fields $\phi^{a}$ can thus be written as

$$
\phi^{a}(\tau, \sigma)=\sum_{n=1}^{\infty} \sqrt{\frac{\alpha^{\prime}}{n}}\left(A_{n}^{\dagger a}(\tau) e^{i n \sigma}+A_{n}^{a}(\tau) e^{-i n \sigma}\right) .
$$

This allows us to compute the equal-time commutator for the $\phi^{a}$ fields:

$$
\left[\phi^{a}\left(\tau, \sigma_{1}\right), \phi^{b}\left(\tau, \sigma_{2}\right)\right]=-2 \pi \alpha^{\prime} i \delta^{a b} \epsilon\left(\sigma_{1}-\sigma_{2}\right),
$$

with

$$
\epsilon(x):=\frac{1}{2 \pi i} \sum_{n \neq 0} \frac{1}{n} e^{i n x},
$$

obeying

$$
\epsilon^{\prime}(x)=\delta(x)-\frac{1}{2 \pi}, \quad \epsilon(x)=-\epsilon(-x) .
$$

This clearly shows that the theory is nonlocal in $\sigma$, given that two fields at separated points do not commute at equal times.

The quantum Hamiltonian can be read off from (4.49) as

$$
H=\frac{1}{2 \alpha^{\prime}} \sum_{n \neq 0} n^{2}: \phi_{a n} \phi_{-n}^{a}:
$$

and allows one to compute the Heisenberg equation:

$$
\dot{\phi}_{n}^{a}=i\left[H, \phi_{n}^{a}\right]=i n \phi_{n}^{a} .
$$

This leads to the on-shell expansion

$$
\phi^{a}(\tau, \sigma)=\sum_{n=1}^{\infty} \sqrt{\frac{\alpha^{\prime}}{n}}\left(A_{n}^{\dagger a} e^{i n x^{+}}+A_{n}^{a} e^{-i n x^{+}}\right),
$$

showing that the spectrum contains purely left-moving massless excitations. With the above expansion one can compute the Feynman propagator: 


$$
\begin{aligned}
\Delta^{a b}(\tau, \sigma) & =\left\langle 0\left|T\left\{\phi^{a}(\tau, \sigma) \phi^{b}(0,0)\right\}\right| 0\right\rangle=\alpha^{\prime} \delta^{a b} \Delta(\tau, \sigma), \\
\Delta(\tau, \sigma) & =\left(\theta(\tau) \sum_{n=1}^{\infty} \frac{1}{n} e^{-i n x^{+}}+\theta(-\tau) \sum_{n=1}^{\infty} \frac{1}{n} e^{i n x^{+}}\right),
\end{aligned}
$$

that can be represented as

$$
\Delta(x)=\frac{i}{4 \pi} \int d^{2} k \frac{e^{i k \cdot x}}{k_{-} k_{1}+i \epsilon},
$$

where we denoted $x^{\alpha}=(\tau, \sigma), k_{\alpha}=(\omega, n)$ and

$$
\int d^{2} k:=\int_{-\infty}^{+\infty} d \omega \sum_{n \neq 0}
$$

The currents (4.39) do not suffer from ordering ambiguities at the quantum level, due to antisymmetrization in the $S O(d)$ indices. We can thus consider $j_{-}^{a b}=0$ and $j_{+}^{a b}=\frac{1}{\alpha^{\prime}} \phi^{[a} \partial_{+} \phi^{b]}$ as quantum operators and use the onshell expansion (4.59) to compute the only nonvanishing two-point function

$$
G_{++}^{a b, c d}(x-y):=\left\langle 0\left|T\left\{j_{+}^{a b}(x) j_{+}^{c d}(y)\right\}\right| 0\right\rangle .
$$

Writing the time-ordered product explicitly we have

$$
\begin{aligned}
G_{++}^{a b, c d}= & \theta\left(\tau_{1}-\tau_{2}\right)\left\langle 0\left|j_{+}^{a b}(x) j_{+}^{c d}(y)\right| 0\right\rangle+\theta\left(\tau_{2}-\tau_{1}\right) \\
& \times\left\langle 0\left|j_{+}^{c d}(y) j_{+}^{a b}(x)\right| 0\right\rangle,
\end{aligned}
$$

where $x^{\alpha}=\left(\tau_{1}, \sigma_{1}\right)$ and $y^{\alpha}=\left(\tau_{2}, \sigma_{2}\right)$. We then focus on the first factor and use the mode expansion to obtain

$$
\begin{aligned}
\left\langle 0\left|j_{+}^{a b}(x) j_{+}^{c d}(y)\right| 0\right\rangle & =\sum_{n, m=1}^{\infty} \sum_{p, q=1}^{\infty} \sqrt{\frac{m q}{n p}}\left\langle 0\left|A_{n}^{[a} A_{m}^{b]} e^{-i(n+m) x^{+}} A_{p}^{\dagger[c} A_{q}^{\dagger d]} e^{i(p+q) y^{+}}\right| 0\right\rangle \\
& =\sum_{n, m=1}^{\infty} \sum_{p, q=1}^{\infty} \sqrt{\frac{m q}{n p}} e^{-i(n+m) x^{+}+i(p+q) y^{+}} \delta^{c[a} \delta^{b] d}\left(\delta_{n p} \delta_{m q}-\delta_{m p} \delta_{n q}\right) \\
& =\frac{1}{2}\left(\delta^{a c} \delta^{b d}-\delta^{b c} \delta^{a d}\right) \sum_{n, m=1}^{\infty}\left(\frac{m}{n}-1\right) e^{-i(n+m)\left(x^{+}-y^{+}\right)} \\
& =\frac{1}{2}\left(\delta^{a c} \delta^{b d}-\delta^{b c} \delta^{a d}\right) F\left(x^{+}-y^{+}\right),
\end{aligned}
$$

where we defined

$$
F(x):=\sum_{n, m=1}^{\infty}\left(\frac{m}{n}-1\right) e^{-i(n+m) x} .
$$

In order to evaluate this function one can perform the sums on $n$ and $m$ separately. By using

$$
\begin{aligned}
\sum_{n=1}^{\infty} z^{n} & =\frac{z}{1-z}, \quad \sum_{n=1}^{\infty} n z^{n}=z \frac{d}{d z} \frac{1}{1-z}=\frac{z}{(1-z)^{2}}, \\
\sum_{n=1}^{\infty} \frac{1}{n} z^{n} & =g(z), \\
\frac{d g}{d z} & =\sum_{n=1}^{\infty} z^{n-1}=\frac{1}{1-z} \rightarrow g(z)=-\log (1-z),
\end{aligned}
$$

one obtains

$F(x)=-\frac{z}{(1-z)^{2}}(z+\log (1-z)), \quad z=e^{-i x}$,

or

$$
F(x)=-\frac{1}{\left(e^{i x}-1\right)^{2}}\left[1+e^{i x} \log \left(1-e^{-i x}\right)\right] .
$$

In order to write the two-point function in momentum space, it is more useful to rewrite the double sum in (4.66) as

$$
\sum_{n, m=1}^{\infty} f(n, m)=\sum_{N=2}^{\infty} \sum_{n=1}^{N-1} f(n, N-n),
$$

with $N=n+m$, yielding ${ }^{6}$

$$
F(x)=\sum_{N=1}^{\infty} f(N) e^{-i N x}
$$

with

$f(N)=\sum_{n=1}^{N-1}\left(\frac{N}{n}-2\right)=N[\psi(N)+\gamma]-2(N-1)$,

\footnotetext{
${ }^{6}$ The sum over $N$ can be extended to $N=1$ since $f(1)=0$.
} 
where $\psi(x)=\frac{\Gamma^{\prime}(x)}{\Gamma(x)}$ is the digamma function and $\gamma$ the Euler-Mascheroni constant. It should be mentioned that rewriting (4.70) is not ambiguous, in that both forms of the series converge to the same function $F(x)$, that is regular for $x \neq 0$.

The full two-point function can thus be written as

$$
\begin{aligned}
G_{++}^{a b, c d}(x)= & \frac{1}{2}\left(\delta^{a c} \delta^{b d}-\delta^{b c} \delta^{a d}\right)\left[\theta(\tau) F\left(x^{+}\right)\right. \\
& \left.+\theta(-\tau) F\left(-x^{+}\right)\right] \\
= & \frac{1}{2}\left(\delta^{a c} \delta^{b d}-\delta^{b c} \delta^{a d}\right) G_{++}(x) .
\end{aligned}
$$

At this point one can use (4.71), together with the integral representation for the step function:

$$
\theta( \pm \tau)= \pm \frac{i}{2 \pi} \int d \omega \frac{e^{-i \omega \tau}}{\omega \pm i \epsilon}
$$

in order to obtain the correlator in momentum space:

$$
\begin{aligned}
G_{++}(p) & :=\int \frac{d^{2} x}{(2 \pi)^{2}} e^{-i p_{\alpha} x^{\alpha}} G_{++}(x) \\
& =\frac{i}{4 \pi p_{-}}\left[p_{1}\left(\psi\left(\left|p_{1}\right|\right)+\gamma\right)-2 p_{1}+2 \operatorname{sign}\left(p_{1}\right)\right],
\end{aligned}
$$

where $\operatorname{sign}(x)$ is the sign function with $\operatorname{sign}(0)=0$.

The above is a straightforward computation of the twopoint functions (that does not require a regularization scheme), establishing that they are nonzero, as it should be for any two-point function of (anti-)Hermitian operators. By the argument around (4.36) this proves that the Ward identities are violated, hence establishing the presence of an anomaly. However, it is not easy to interpret the corresponding effective action and hence to compute its (anomalous) transformation. We therefore turn to the more conventional Feynman diagram computation that does require a regularization scheme. As is customary also for chiral fermions we change gears by doing the computation on the plane, as opposed to the cylinder, which has the advantage that one regains Lorentz invariance. According to general lore, an anomaly does not depend on the topology [39], and so this should not affect the invariant result.

We shall thus consider the action (4.37) on the plane and couple it to two-dimensional gauge fields $A_{\alpha}^{a b}$, promoting $S O(d)$ to a local symmetry:

$$
S[\phi, A]=\frac{1}{2 \pi \alpha^{\prime}} \int d^{2} x D_{1} \phi^{a} D_{-} \phi_{a},
$$

where $D_{\alpha} \phi^{a}:=\partial_{\alpha} \phi^{a}+A_{\alpha}^{a b} \phi_{b}$ and under a local $S O(d)$ rotation $\delta_{\lambda} A_{\alpha}^{a b}=-D_{\alpha} \lambda^{a b}$. In order to keep track of index contractions, it is useful to introduce the matrix

$$
g^{\alpha \beta}:=\left(\begin{array}{ll}
0 & 1 \\
1 & 0
\end{array}\right), \quad \text { where } \alpha=(-, 1),
$$

so that the above action can be recast in the form

$$
S=\frac{1}{4 \pi \alpha^{\prime}} \int d^{2} x g^{\alpha \beta} D_{\alpha} \phi^{a} D_{\beta} \phi_{a}
$$

that allows one to write the cubic and quartic vertices as

$$
\begin{aligned}
& S_{3}=-\frac{1}{2 \pi \alpha^{\prime}} \int d^{2} x g^{\alpha \beta} A_{\alpha}^{a b} \phi_{a} \partial_{\beta} \phi_{b}, \\
& S_{4}=\frac{1}{4 \pi \alpha^{\prime}} \int d^{2} x g^{\alpha \beta} A_{\alpha}^{c a} A_{\beta c}{ }^{b} \phi_{a} \phi_{b} .
\end{aligned}
$$

We define the one-loop effective action for $A_{\alpha}^{a b}$ by

$$
e^{i W[A]}=Z^{-1} \int D \phi e^{i S[\phi, A]}=\left\langle e^{i\left(S_{3}+S_{4}\right)}\right\rangle,
$$

where $Z$ is the free $\phi$-path integral normalization and we denote averages by $\langle\ldots\rangle$. We focus on the quadratic part of $W[A]$, that is given by

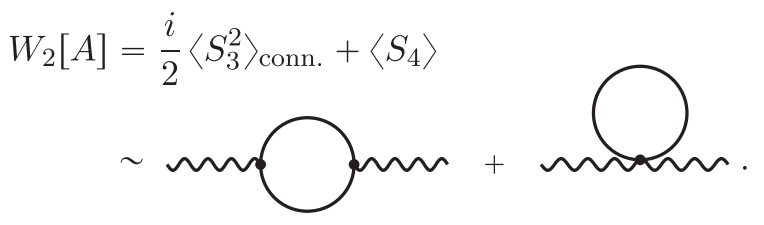

By using the propagator

$$
\left\langle\phi^{a}(x) \phi^{b}(y)\right\rangle=\frac{i \alpha^{\prime}}{4 \pi} \delta^{a b} \int d^{2} k \frac{e^{i k \cdot(x-y)}}{k_{-} k_{1}+i \epsilon}
$$

and the Fourier representation

$$
A_{\alpha}^{a b}(x)=\int d^{2} k e^{i k \cdot x} A_{\alpha}^{a b}(k)
$$

the effective action $W_{2}[A]$ can be written in the form

$W_{2}[A]=-\frac{i}{4} \int d^{2} p g^{\alpha \gamma} g^{\beta \delta} A_{\gamma}^{a b}(p) \Pi_{\alpha \beta}(p) A_{\delta a b}(-p)$.

The polarization tensor $\Pi_{\alpha \beta}(p)$ reads 


$$
\Pi_{\alpha \beta}(p)=-\frac{1}{2} \int d k_{-} d k_{1} \frac{\left(2 k_{\alpha}-p_{\alpha}\right)\left(2 k_{\beta}-p_{\beta}\right)-2 g_{\alpha \beta}\left[k_{-} k_{1}+\left(p_{-}-k_{-}\right)\left(p_{1}-k_{1}\right)\right]}{k_{1}\left(p_{1}-k_{1}\right)\left(k_{-}+\frac{i \epsilon}{k_{1}}\right)\left(k_{-}-p_{-}-\frac{i \epsilon}{p_{1}-k_{1}}\right)}
$$

where the integrand has manifest symmetry in the exchange $k_{\alpha} \leftrightarrow\left(p_{\alpha}-k_{\alpha}\right)$.

We regularize the integral over $k_{-}$by inserting a factor $e^{i \delta k_{-} k_{1}}$. This allows us to compute the integral by closing the contour in the complex $k_{-}$plane. Although this regulator is not Lorentz invariant, it does not affect the anomaly. The remaining integral over $k_{1}$ suffers from infrared divergences, appearing as $\int_{0}^{\left|p_{1}\right|} \frac{d k_{1}}{k_{1}}$. This is to be expected from a two-dimensional massless scalar on the infinite plane, while this divergence disappears on the cylinder, due to the discrete spatial momentum. We will thus regulate the $k_{1}$ integral by introducing an infrared cutoff $\mu$, substituting $\int_{0}^{\left|p_{1}\right|}$ with $\int_{\mu}^{\left|p_{1}\right|}$. The various components of the polarization tensor read

$$
\begin{aligned}
\Pi_{11}(p) & =-2 \pi i \frac{p_{1}}{p_{-}}\left[\log \left(\left|p_{1}\right| / \mu\right)-2\right], \\
\Pi_{--}(p) & =-2 \pi i \frac{p_{-}}{p_{1}} \log \left(\left|p_{1}\right| / \mu\right), \\
\Pi_{-1}(p) & =2 \pi i \log \left(\left|p_{1}\right| / \mu\right),
\end{aligned}
$$

yielding the effective action

$$
\begin{aligned}
W_{2}[A]= & -\frac{\pi}{2} \int d^{2} p\left\{A_{-}^{a b}(p) \frac{p_{1}}{p_{-}}\left[\log \left(\left|p_{1}\right| / \mu\right)-2\right] A_{-a b}(-p)\right. \\
& +A_{1}^{a b}(p) \frac{p_{-}}{p_{1}} \log \left(\left|p_{1}\right| / \mu\right) A_{1 a b}(-p) \\
& \left.-2 A_{1}^{a b}(p) \log \left(\left|p_{1}\right| / \mu\right) A_{-a b}(-p)\right\}
\end{aligned}
$$

To recast the above expression in the more familiar lightcone basis one can substitute $A_{1}^{a b}=A_{+}^{a b}-A_{-}^{a b}$ and similarly $p_{1}=p_{+}-p_{-}$. Before computing the gauge variation of (4.87), we shall notice that the term $A_{-}^{a b} \frac{p_{1}}{p_{-}} A_{-a b}$ differs from $A_{-}^{a b} \frac{p_{+}}{p_{-}} A_{-a b}$ by a purely local term, implying that one can use the latter to compute the anomaly.

To lowest order in $A_{\alpha}^{a b}$ one has $\delta_{\lambda} A_{\alpha}^{a b}(p)=-i p_{\alpha} \lambda^{a b}(p)$, finally yielding the anomalous variation

$$
\delta_{\lambda} W_{2}[A]=-2 \pi i \int d^{2} p \lambda^{a b}(p) p_{+} A_{-a b}(-p)+\mathcal{O}\left(A^{2}\right)
$$

that is Lorentz invariant and structurally the same as the one due to a chiral fermion. As usual, in order to establish that (4.88) is a genuine anomaly, one has to consider adding to the effective action all possible local counterterms. Since
(4.88) is Lorentz invariant, the only local counterterm that can change it is

$$
\Delta W_{2}[A]=\alpha \int d^{2} p A_{+}^{a b}(p) A_{-a b}(-p),
$$

leading to

$$
\begin{aligned}
\delta_{\lambda}\left(W_{2}+\Delta W_{2}\right)= & -i \int d^{2} p \lambda^{a b}(p)\left[(2 \pi+\alpha) p_{+} A_{-a b}(-p)\right. \\
& \left.+\alpha p \_A_{+a b}(-p)\right] .
\end{aligned}
$$

The above result shows that no value of $\alpha$ can make the effective action gauge invariant, thus establishing that (4.88) is a genuine anomaly. We shall choose the value $\alpha=-\pi$, in order to have a purely parity-violating anomalous variation. Defining

$$
W_{2}^{\mathrm{eff}}[A]:=W_{2}[A]-\pi \int d^{2} p A_{+}^{a b}(p) A_{-a b}(-p),
$$

with $W_{2}[A]$ given by (4.87), we finally obtain, using $\epsilon^{+-}=2$,

$$
\begin{aligned}
\delta_{\lambda} W_{2}^{\mathrm{eff}}[A] & =-i \pi \int d^{2} p \lambda^{a b}(p)\left[p_{+} A_{-a b}(-p)-p_{-} A_{+a b}(-p)\right] \\
& =\frac{1}{4 \pi} \int d^{2} x \lambda_{a b}\left[\partial_{+} A_{-}^{a b}-\partial_{-} A_{+}^{a b}\right] \\
& =\frac{1}{8 \pi} \int d^{2} x \lambda_{a b} \epsilon^{\alpha \beta} \partial_{\alpha} A_{\beta}^{a b} .
\end{aligned}
$$

\section{Green-Schwarz deformation}

After computing the $S O(d)$ anomaly due to left-moving Floreanini-Jackiw bosons we can now apply the result to the worldsheet sigma model (3.20). The action for the "internal" $Y$ sector (4.20) reads

$$
\begin{aligned}
S_{Y}= & \frac{1}{2 \pi \alpha^{\prime}} \int d^{2} \sigma\left[\left(\mathcal{D}_{1} Y^{\underline{A}}+P_{1} \underline{A}_{\bar{B}} Y^{\bar{B}}\right)\left(\mathcal{D}_{-} Y_{\underline{A}}+P_{-\underline{A} \bar{C}} Y^{\bar{C}}\right)\right. \\
& \left.+\left(\mathcal{D}_{1} Y^{\bar{A}}+P_{1}{ }_{\underline{B}}^{\bar{A}} Y^{\underline{B}}\right)\left(\mathcal{D}_{+} Y_{\bar{A}}+P_{+\bar{A}} \underline{C} Y^{\underline{C}}\right)\right],
\end{aligned}
$$

with $\mathcal{D}_{\alpha} Y^{\underline{A}}=\partial_{\alpha} Y^{\underline{A}}+Q_{\bar{\alpha}}^{A B} Y_{\underline{B}}, \quad \mathcal{D}_{\alpha} Y^{\bar{A}}=\partial_{\alpha} Y^{\bar{A}}+Q_{\alpha}^{\overline{A B}} Y_{\bar{B}}$ and we recall that $Q_{\alpha}=\partial_{\alpha} X^{\mu} Q_{\mu}$ and $P_{\alpha}=\partial_{\alpha} X^{\mu} P_{\mu}$ are the pullbacks of the background fields.

Our goal is to investigate the one-loop effective action (still depending on the $X^{\mu}$ worldsheet fields and background fields $Q_{\mu}$ and $P_{\mu}$ ) generated by integrating out the internal fields $\left(Y^{A}, Y^{\bar{A}}\right)$. We first focus on the case 
$P_{\bar{\alpha}}^{A} \bar{B}=0$, where one can see that the above action reduces to the sum of a left-moving FJ action (4.76) with gauged $S O(d)_{L}$ and an analogous right-moving action with gauged $S O(d)_{R}$. By just replacing $\phi^{a} \rightarrow Y^{A}, A_{\alpha}^{a b} \rightarrow Q_{\alpha}^{A B}$ one obtains an effective action $W_{2}[Q, \bar{Q}]$ whose anomalous $S O(d)_{L} \times S O(d)_{R}$ variation is given, to lowest order, by

$$
\begin{aligned}
\delta_{\lambda, \bar{\lambda}} W_{2}[Q, \bar{Q}]= & \frac{1}{8 \pi} \int d^{2} x \lambda_{\underline{A B}} \epsilon^{\alpha \beta} \partial_{\alpha} Q_{\bar{\beta}}^{\underline{A B}} \\
& -\frac{1}{8 \pi} \int d^{2} x \lambda_{\overline{A B}} \epsilon^{\alpha \beta} \partial_{\alpha} Q_{\beta}^{\overline{A B}},
\end{aligned}
$$

where the right-moving contribution can be obtained by a computation analogous to the one presented in the previous subsection.

We can now examine the effect of the $P_{\alpha}$ tensor on the full effective action $W_{2}[Q, \bar{Q}, P]$. Due to the orthogonality of the two $S O(d)$ groups, the only contribution of $P_{\alpha}$ to the quadratic effective action $W_{2}$ has to be of the form

$$
\int d^{2} k P_{\alpha}^{A} \bar{B}(k) G^{\alpha \beta}(k) P_{\beta \underline{A} \bar{B}}(-k) .
$$

Since the gauge transformation of the $P$ tensor is $\delta_{\lambda} P_{\mu \underline{A} \bar{B}}=$ $\lambda_{\underline{A}} \underline{C} P_{\mu \underline{C} \bar{B}}+\lambda_{\bar{B}} \bar{C} P_{\mu \underline{A} \bar{C}}$, the variation of (4.95), if nonvanishing, cannot contribute to linear order in the background fields. This shows that, to lowest order in the fields, the anomalous variation of $W[Q, \bar{Q}, P]$ is given by (4.94), that can be written in form language as

$\delta_{\lambda, \bar{\lambda}} W[Q, \bar{Q}, P]=\frac{1}{8 \pi} \int \operatorname{tr}(d \lambda \wedge Q)-\frac{1}{8 \pi} \int \operatorname{tr}(d \bar{\lambda} \wedge \bar{Q})$.

Since the above result already satisfies the Wess-Zumino consistency conditions $\left[\delta_{\lambda_{1}}, \delta_{\lambda_{2}}\right] W=\delta_{\left[\lambda_{2}, \lambda_{1}\right]} W$, (4.96) does not receive higher order contributions in $Q$ and $P$, and gives the full anomaly. Let us mention that in cosmological settings, where the "external" coordinates $X^{\mu}$ reduce to time $X^{0}=t$, one has $d Q^{a b}=d \sigma^{\alpha} \wedge d \sigma^{\beta} \partial_{\alpha} t \partial_{\beta} t \partial_{t} Q_{t}^{a b} \equiv 0$ and the anomaly is not present.

The above anomalous variation, if not canceled, implies that gauge-equivalent background fields (from the target space perspective) lead to inequivalent worldsheet sigma models, which is not acceptable. Fortunately, in the same spirit of the original Green-Schwarz mechanism [29], the anomaly (4.96) can be canceled by postulating a suitable transformation for the $B$-field. We recall from (3.20) that the action involving the Kalb-Ramond field reads

$$
S_{B}=-\frac{1}{4 \pi \alpha^{\prime}} \int d^{2} x \epsilon^{\alpha \beta} B_{\alpha \beta},
$$

where $B_{\alpha \beta}$ denotes the pullback of $B_{\mu \nu}$. At this point, simple inspection of (4.96) determines that the anomaly can be canceled by assigning to the $B$-field the transformation law

$$
\delta_{\lambda, \bar{\lambda}} B=\frac{\alpha^{\prime}}{2} \operatorname{tr}(d \lambda \wedge Q)-\frac{\alpha^{\prime}}{2} \operatorname{tr}(d \bar{\lambda} \wedge \bar{Q}) .
$$

Remarkably, this is exactly the transformation found in [19] from the low-energy target space analysis, thus showing that its emergence in the worldsheet theory stems from the anomalies of two-dimensional chiral bosons.

As a final comment, the form (4.96) of the $S O(d)_{L} \times$ $S O(d)_{R}$ anomaly shows that the diagonal $S O(d)$ subgroup remains unbroken. This also agrees with the analysis of [19] and is to be expected, since the diagonal $S O(d)$ is the geometric subgroup of $S O(d)_{L} \times S O(d)_{R}$.

\section{CONCLUSIONS AND OUTLOOK}

In this paper we have revisited the issue of making the T-duality group $O(d, d)$ a manifest symmetry of the worldsheet action of (bosonic) string theory. We have identified a consistent truncation with global $O(d, d, \mathbb{R})$ invariance, in which the target space fields are independent of $d$ coordinates while the worldsheet scalars have zero (internal) momentum and winding. This truncation may be thought of as the zero-mass sector for a Kaluza-Klein compactification on a $d$-dimensional torus, but the topology is no longer relevant-precisely because of the truncation to zero momentum and winding. As such, this worldsheet theory is applicable to any setting with $d$ Abelian isometries, be they compact or not, in particular to cosmological backgrounds, as employed in [40]. We have displayed the proper manifestly $O(d, d, \mathbb{R})$ invariant worldsheet action that includes all target space fields that survive the truncation.

As the second main point of this paper we have shown that the $S O(d)_{L} \times S O(d)_{R}$ local frame transformations are anomalous, as to be expected given the presence of chiral bosons. This suggests that a Green-Schwarz mechanism is needed in which the (external) $B$-field, which is a singlet in the classical theory, transforms nontrivially under these symmetries, in line with recent findings in the target space theory when higher oder $\alpha^{\prime}$ corrections are included $[19,20]$. This result has a direct bearing on any attempts to determine the target space equations directly in $O(d, d, \mathbb{R})$ invariant form by computing the beta functions of a suitable $O(d, d, \mathbb{R})$ invariant worldsheet theory, a program that was initiated in [10-12]. It will then be important to revisit this program in light of the present results, in particular to develop precise computational rules that allow one, in principle, to determine the equations to arbitrary orders in $\alpha^{\prime}$. In this respect, one of the main difficulties in using this formalism is the lack of manifest two-dimensional Lorentz invariance. It would then be interesting to investigate the proposal, made in [2], of 
modifying the functional measure of the chiral bosons as $D \phi \rightarrow D \phi\left(\operatorname{det} \partial_{1}\right)^{1 / 2}$. This formally relates the path integral of the Floreanini-Jackiw bosons to the one of chiral fermions, which is manifestly Lorentz invariant.

A manifestly $O(d, d, \mathbb{R})$ invariant procedure to compute the beta functions may be particularly fruitful in the cosmological setting, which is significantly simplified since the external dimensions are reduced to (cosmic) time, and where a complete classification of all duality invariant $\alpha^{\prime}$ corrections has been found recently [40]. It remains to fix a finite number of free parameters at each order in $\alpha^{\prime}$, and one may hope that this could eventually be achieved by a worldsheet computation using the results given here.

Let us finally mention that while the general phenomenon for which we provide here a worldsheet interpretation was first discovered in double field theory $[21,23]$, none of our findings depend directly on double field theory. They are a feature of a standard string theory formulation. Nevertheless, the most enticing extension of this framework would of course be to a full-fledged double field theory. In the truncation invoked here there is a clear separation of dimensions along which the fields may vary (external) and of dimensions along which the fields are constant (internal), with the $O(d, d, \mathbb{R})$ acting exclusively on the latter. This truncation is explicitly $O(d, d, \mathbb{R})$ invariant to all orders in $\alpha^{\prime}$, as follows by general arguments [41] and explicit computations [40,42], and so there should be a worldsheet conformal field theory construction giving these target space equations. However, a genuine double field theory would go beyond this by having fields that in addition depend on doubled internal coordinates, corresponding to the scalar fields $Y^{M}$, subject to the level-matching constraint (that now does assume a torus background) and obeying a novel algebra [43]. The results obtained here may help to illuminate some issues that arise when trying to define this theory explicitly.

\section{ACKNOWLEDGMENTS}

We would like to thank Fiorenzo Bastianelli, Chris Blair, Ashoke Sen, and Arkady Tseytlin for helpful discussions. This work is supported by the ERC Consolidator Grant No. 771862 "Symmetries and Cosmology."

\section{APPENDIX A: WORLDSHEET DIFFEOMORPHISMS}

Here we derive various nonstandard realizations of worldsheet diffeomorphisms, which in our formulation are not manifest. In particular, we show that in the Hamiltonian picture they are generated by the Virasoro constraints, as to be expected. More importantly, we also show that the $\tilde{Y}_{i}$, introduced by a nonlocal field redefinition $2 \pi \alpha^{\prime} P_{i}=\partial_{\sigma} \tilde{Y}_{i}$, admit local diffeomorphism transformations. Finally, we show that diffeomorphism invariance, despite its nonstandard realization, implies energy-momentum conservation in the standard form.

\section{Equivalence between Lagrangian and Hamiltonian diffeomorphisms}

Let us start from the sigma model action (3.5), i.e.,

$$
\begin{aligned}
S_{L}= & -\frac{1}{4 \pi \alpha^{\prime}} \int d^{2} \sigma\left[\sqrt{-h} h^{\alpha \beta} \hat{G}_{\hat{\mu} \hat{\nu}}(X)\right. \\
& \left.+\epsilon^{\alpha \beta} \hat{B}_{\hat{\mu} \hat{\nu}}(X)\right] \partial_{\alpha} \hat{X}^{\hat{\mu}} \partial_{\beta} \hat{X}^{\hat{\nu}},
\end{aligned}
$$

where here and in the following the subscript $L$ refers to "Lagrangian," as opposed to $H$ which will refer to "Hamiltonian." Diffeomorphism and Weyl transformations take the familiar form

$$
\begin{aligned}
\delta_{L} h_{\alpha \beta} & =\xi^{\lambda} \partial_{\lambda} h_{\alpha \beta}+2 \partial_{(\alpha} \xi^{\lambda} h_{\beta) \lambda}+2 \omega h_{\alpha \beta}, \\
\delta_{L} \hat{X}^{\hat{\mu}} & =\xi^{\alpha} \partial_{\alpha} \hat{X}^{\hat{\mu}} .
\end{aligned}
$$

Using the parametrization (2.5) for the metric one finds

$$
\begin{aligned}
\delta_{L} \Omega & =\partial_{\alpha}\left(\Omega \xi^{\alpha}\right)+2 \omega \Omega, \\
\delta_{L} e & =\xi^{\alpha} \partial_{\alpha} e+e\left[\partial_{\tau} \xi^{\tau}-\partial_{\sigma} \xi^{\sigma}-2 u \partial_{\sigma} \xi^{\tau}\right], \\
\delta_{L} u & =\xi^{\alpha} \partial_{\alpha} u+\partial_{\tau} \xi^{\sigma}+u\left[\partial_{\tau} \xi^{\tau}-\partial_{\sigma} \xi^{\sigma}\right]-\partial_{\sigma} \xi^{\tau}\left[u^{2}+e^{2}\right],
\end{aligned}
$$

which is awkward, since the basis $(e, u, \Omega)$ is adapted to the Hamiltonian formulation.

We will now determine the Hamiltonian form of the diffeomorphisms, which are generated via Poisson brackets from the Virasoro constraints. The Hamiltonian action associated to (A1) was given in (2.6), except that now we would have to replace all indices by hatted indices, referring to the totality of internal and external components. However, here we will not be concerned with the split into "external" $X^{\mu}$ and "internal" $Y^{i}$, and so in order not to overburden the notation, we shall drop all hats from our formulas in what follows. In particular, $X^{\mu}$ and $P_{\mu}$ stand for $(n+d)$-dimensional phase space variables, $G_{\mu \nu}$ and $B_{\mu \nu}$ denote the spacetime metric and $B$-field in $(n+d)$ dimensions and, finally, capital indices $M=1, \ldots, 2(d+n)$ denote $O(d+n, d+n)$ tensors.

The fundamental Poisson brackets are

$$
\left\{X^{\mu}\left(\sigma_{1}\right), P_{\nu}\left(\sigma_{2}\right)\right\}=\delta_{\nu}^{\mu} \delta\left(\sigma_{1}-\sigma_{2}\right) .
$$

The simple form of the constraints in terms of $O(D, D)$ quantities in (2.10) suggests to use the covariant Poisson brackets

$$
\left\{Z^{M}\left(\sigma_{1}\right), Z^{N}\left(\sigma_{2}\right)\right\}=2 \pi \alpha^{\prime} \eta^{M N} \partial_{\sigma_{1}} \delta\left(\sigma_{1}-\sigma_{2}\right),
$$

along with 


$$
\left\{Z_{M}\left(\sigma_{1}\right), \Phi\left(X\left(\sigma_{2}\right)\right)\right\}=-2 \pi \alpha^{\prime} \partial_{M} \Phi(X) \delta\left(\sigma_{1}-\sigma_{2}\right)
$$

for $X$-dependent fields, where it is understood that $\tilde{\partial}^{\mu} \Phi(X) \equiv 0$. Using (A5) and (A6) it is indeed simple to compute the local constraint algebra:

$$
\begin{aligned}
& \left\{\mathcal{N}\left(\sigma_{1}\right), \mathcal{N}\left(\sigma_{2}\right)\right\}=\frac{1}{2 \pi \alpha^{\prime}} Z^{M}\left(\sigma_{1}\right) Z_{M}\left(\sigma_{2}\right) \partial_{\sigma_{1}} \delta\left(\sigma_{1}-\sigma_{2}\right) \\
& \left\{\mathcal{N}\left(\sigma_{1}\right), \mathcal{H}\left(\sigma_{2}\right)\right\}=\frac{1}{2 \pi \alpha^{\prime}} Z^{M}\left(\sigma_{1}\right) Z^{N}\left(\sigma_{2}\right) \mathcal{H}_{M N}\left(\sigma_{2}\right) \partial_{\sigma_{1}} \delta\left(\sigma_{1}-\sigma_{2}\right)-\frac{1}{4 \pi \alpha^{\prime}} Z^{P}\left(\sigma_{1}\right) \partial_{P} \mathcal{H}_{M N}\left(\sigma_{1}\right) Z^{M}\left(\sigma_{1}\right) Z^{N}\left(\sigma_{1}\right) \delta\left(\sigma_{1}-\sigma_{2}\right) \\
& \left\{\mathcal{H}\left(\sigma_{1}\right), \mathcal{H}\left(\sigma_{2}\right)\right\}=\frac{1}{2 \pi \alpha^{\prime}} Z^{M}\left(\sigma_{1}\right) \mathcal{H}_{M P}\left(\sigma_{1}\right) \mathcal{H}^{P}{ }_{N}\left(\sigma_{2}\right) Z^{N}\left(\sigma_{2}\right) \partial_{\sigma_{1}} \delta\left(\sigma_{1}-\sigma_{2}\right)
\end{aligned}
$$

where we can reduce $Z^{P} \partial_{P} \mathcal{H}_{M N}=\partial_{\sigma} X^{\mu} \partial_{\mu} \mathcal{H}_{M N}=\partial_{\sigma} \mathcal{H}_{M N}$. Given the local form (A7), the constraint algebra, adopting the formalism in Refs. $[14,44]$, is most easily read in terms of the smeared constraints

$$
N(\alpha):=\int_{0}^{2 \pi} d \sigma \alpha(\sigma) \mathcal{N}(\sigma), \quad H(\epsilon):=\int_{0}^{2 \pi} d \sigma \epsilon(\sigma) \mathcal{H}(\sigma),
$$

for which the first-class property becomes apparent:

$$
\begin{gathered}
\left\{N\left(\alpha_{1}\right), N\left(\alpha_{2}\right)\right\}=N\left(\left[\alpha_{1}, \alpha_{2}\right]\right), \\
\{N(\alpha), H(\epsilon)\}=H([\alpha, \epsilon]), \\
\left\{H\left(\epsilon_{1}\right), H\left(\epsilon_{2}\right)\right\}=N\left(\left[\epsilon_{1}, \epsilon_{2}\right]\right),
\end{gathered}
$$

with square brackets denoting one-dimensional Lie brackets, i.e.,

$$
\left[\alpha_{1}, \alpha_{2}\right]:=\alpha_{1} \partial_{\sigma} \alpha_{2}-\alpha_{2} \partial_{\sigma} \alpha_{1}
$$

One can choose a diagonal basis for $\mathcal{N}$ and $\mathcal{H}$ by using the projectors $\Pi_{M N}^{ \pm}:=\frac{1}{2}\left(\eta_{M N} \pm \mathcal{H}_{M N}\right)$ [which can be quickly verified to be projectors thanks to $\mathcal{H}_{M N}$ being an $O(D, D)$ element]:

$$
\mathcal{H}_{ \pm}:=\frac{1}{2}(\mathcal{N} \pm \mathcal{H})=\frac{1}{4 \pi \alpha^{\prime}} \Pi_{M N}^{ \pm} Z^{M} Z^{N}
$$

The Hamiltonian algebra (A9) then takes the manifest $\mathfrak{D i f f}_{1} \oplus \mathfrak{d i f f}_{1}$ form:

$$
\begin{aligned}
\left\{H_{ \pm}\left(\alpha_{1}\right), H_{ \pm}\left(\alpha_{2}\right)\right\} & =H_{ \pm}\left(\left[\alpha_{1}, \alpha_{2}\right]\right), \\
\left\{H_{+}(\alpha), H_{-}(\beta)\right\} & =0 .
\end{aligned}
$$

The smeared constraints (A8) can be used to determine the Hamiltonian gauge transformations of the phase space fields. Taking Poisson brackets one finds

$$
\begin{aligned}
\delta_{H} X^{\mu} & =\left\{X^{\mu}, N(\alpha)+H(\epsilon)\right\}=\alpha \partial_{\sigma} X^{\mu}+\epsilon \mathcal{H}^{\mu}{ }_{N} Z^{N}, \\
\delta_{H} P_{\mu} & =\left\{P_{\mu}, N(\alpha)+H(\epsilon)\right\} \\
& =\partial_{\sigma}\left[\alpha P_{\mu}+\frac{1}{2 \pi \alpha^{\prime}} \epsilon \mathcal{H}_{\mu N} Z^{N}\right]-\frac{1}{4 \pi \alpha^{\prime}} \epsilon \partial_{\mu} \mathcal{H}_{M N} Z^{M} Z^{N},
\end{aligned}
$$

that are the Hamiltonian version of $\mathfrak{d} \mathfrak{i} \mathfrak{i f}_{2}$ transformations. Finally, requiring invariance of the Hamiltonian action under the above local transformations determines the transformation law of the corresponding Hamiltonian gauge fields $e$ and $u$ :

$$
\delta_{H} e=\partial_{\tau} \epsilon-[u, \epsilon]-[e, \alpha], \quad \delta_{H} u=\partial_{\tau} \alpha-[u, \alpha]-[e, \epsilon],
$$

where again square brackets denote one-dimensional Lie brackets.

The transformation laws (A13) and (A14) have to be compared with their Lagrangian counterpart (A3) and $\delta_{L} X^{\mu}=\xi^{\alpha} \partial_{\alpha} X^{\mu}$. Defining the Lagrangian transformation $\delta_{L} P_{\mu}$ for momenta may seem counterintuitive, but it is simply determined by considering the transformation of $P_{\mu}^{\mathrm{lag}} \equiv \frac{\partial \mathcal{L}}{\partial \dot{X}^{\mu}}$ as a given function of Lagrangian variables, i.e.,

$P_{\mu}^{\mathrm{lag}}=\frac{1}{2 \pi \alpha^{\prime}}\left[\frac{1}{e} G_{\mu \nu}\left(\partial_{\tau} X^{\nu}-u \partial_{\sigma} X^{\nu}\right)+B_{\mu \nu} \partial_{\sigma} X^{\nu}\right]$,

that, obviously, is just the on-shell value of the Hamiltonian momentum. Using the transformation law of $P_{\mu}^{\mathrm{lag}}$ to define $\delta_{L} P_{\mu}$ ensures that such transformations commute with integrating out momenta.

The simplest way to determine the transformation law of $P_{\mu}^{\mathrm{lag}}$ is to view it as the $\tau$ component of the two-dimensional vector density

$$
\pi_{\mu}^{\alpha}:=-\frac{1}{2 \pi \alpha^{\prime}}\left[\sqrt{-h} h^{\alpha \beta} G_{\mu \nu} \partial_{\beta} X^{\nu}+\epsilon^{\alpha \beta} B_{\mu \nu} \partial_{\beta} X^{\nu}\right],
$$

that transforms as 


$$
\delta_{L} \pi_{\mu}^{\alpha}=\partial_{\beta}\left(\xi^{\beta} \pi_{\mu}^{\alpha}\right)-\partial_{\beta} \xi^{\alpha} \pi_{\mu}^{\beta}
$$

thus yielding

$\delta_{L} X^{\mu}=\xi^{\alpha} \partial_{\alpha} X^{\mu}$,

$\delta_{L} P_{\mu}=\xi^{\tau} \partial_{\tau} P_{\mu}+\partial_{\sigma}\left(\xi^{\sigma} P_{\mu}\right)+\partial_{\sigma} \xi^{\tau}\left(u P_{\mu}+\frac{1}{2 \pi \alpha^{\prime}} e \mathcal{H}_{\mu N} Z^{N}\right)$.

At this point, the final ingredient to prove equivalence of the Lagrangian $\mathbf{D} i \tilde{f f}_{2}$ transformations (A18) with the canonical ones (A13), are the Hamiltonian field equations

$$
\begin{aligned}
\frac{\delta S_{H}}{\delta P_{\mu}}= & \partial_{\tau} X^{\mu}-u \partial_{\sigma} X^{\mu}-e \mathcal{H}^{\mu}{ }_{N} Z^{N}, \\
\frac{\delta S_{H}}{\delta X^{\mu}}= & -\partial_{\tau} P_{\mu}+\partial_{\sigma}\left[u P_{\mu}+\frac{1}{2 \pi \alpha^{\prime}} e \mathcal{H}_{\mu N} Z^{N}\right] \\
& -\frac{e}{4 \pi \alpha^{\prime}} \partial_{\mu} \mathcal{H}_{M N} Z^{M} Z^{N} .
\end{aligned}
$$

Inspection of the transformation laws (A13) and (A18) allows us to determine the relation between the canonical gauge parameters $(\alpha, \epsilon)$ and the geometric vector field $\xi^{\alpha}$, namely

$$
\epsilon=e \xi^{\tau}, \quad \alpha=\xi^{\sigma}+u \xi^{\tau}
$$

With the above redefinition we can finally establish the explicit equivalence between two-dimensional diffeomorphisms and canonical gauge transformations as

$$
\begin{aligned}
\delta_{H} X^{\mu} & =\delta_{L} X^{\mu}-\xi^{\tau} \frac{\delta S_{H}}{\delta P_{\mu}}, \quad \delta_{H} P_{\mu}=\delta_{L} P_{\mu}+\xi^{\tau} \frac{\delta S_{H}}{\delta X^{\mu}}, \\
\delta_{H} e & =\delta_{L} e, \quad \delta_{H} u=\delta_{L} u .
\end{aligned}
$$

The extra terms in $\delta_{H} X^{\mu}$ and $\delta_{H} P_{\mu}$ are indeed of the trivial form $\delta \varphi^{i}=\mu^{i j} \frac{\delta S}{\delta \varphi^{j}}$ with $\mu^{i j}$ antisymmetric. This kind of local transformations is not related to any genuine gauge redundancy, and can be safely ignored.

\section{Locality and $\boldsymbol{O}(\boldsymbol{d}, \boldsymbol{d})$ invariance of diffeomorphisms}

After proving equivalence of Lagrangian diffeomorphisms (A18) and Hamiltonian gauge transformations (A13) for the general sigma model in $(n+d)$ dimensions, we shall now study the split $\hat{X}^{\hat{\mu}}=\left(X^{\mu}, Y^{i}\right)$ between external and internal sectors.

First of all, let us reinstate the original notation, with hatted symbols denoting $(n+d)$-dimensional fields, and rewrite the Lagrangian and Hamiltonian transformations:

$$
\begin{aligned}
\delta_{L} \hat{X}^{\hat{\mu}}= & \xi^{\alpha} \partial_{\alpha} \hat{X}^{\hat{\mu}} \\
\delta_{L} \hat{P}_{\hat{\mu}}= & \xi^{\tau} \partial_{\tau} \hat{P}_{\hat{\mu}}+\partial_{\sigma}\left(\xi \sigma \hat{P}_{\hat{\mu}}\right) \\
& +\partial_{\sigma} \xi^{\tau}\left(u \hat{P}_{\hat{\mu}}+\frac{1}{2 \pi \alpha^{\prime}} e \hat{\mathcal{H}}_{\hat{\mu} \hat{N}} \hat{Z}^{\hat{N}}\right),
\end{aligned}
$$

and

$$
\begin{aligned}
& \delta_{H} \hat{X}^{\hat{\mu}}=\alpha \partial_{\sigma} \hat{X}^{\hat{\mu}}+\epsilon \hat{\mathcal{H}}^{\hat{\mu}} \hat{N}^{\hat{N}}, \\
& \delta_{H} \hat{P}_{\hat{\mu}}=\partial_{\sigma}\left[\alpha \hat{P}_{\hat{\mu}}+\frac{1}{2 \pi \alpha^{\prime}} \epsilon \hat{\mathcal{H}}_{\hat{\mu} \hat{N}} \hat{Z}^{\hat{N}}\right]-\frac{1}{4 \pi \alpha^{\prime}} \epsilon \partial_{\hat{\mu}} \hat{\mathcal{H}}_{\hat{M} \hat{N}} \hat{Z}^{\hat{M}} \hat{Z}^{\hat{N}} .
\end{aligned}
$$

Let us also remind the reader that the $O(d+n, d+n)$ vector $\hat{Z}^{\hat{M}}$ is given by

$$
\hat{Z}^{\hat{M}}=\left(\begin{array}{c}
\partial_{\sigma} \hat{X}^{\hat{\mu}} \\
2 \pi \alpha^{\prime} \hat{P}_{\hat{\mu}}
\end{array}\right),
$$

and the $O(d+n, d+n)$ generalized metric is defined in terms of $\hat{G}_{\hat{\mu} \hat{\nu}}$ and $\hat{B}_{\hat{\mu} \hat{\nu}}$.

Upon splitting the phase space variables as $\hat{X}^{\hat{\mu}}=$ $\left(X^{\mu}, Y^{i}\right)$ and $\hat{P}_{\hat{\mu}}=\left(P_{\mu}, P_{i}\right)$, we recall that our action (3.20) is purely Lagrangian in the noncompact sector. The diffeomorphism transformations thus act as usual: $\delta X^{\mu}=\xi^{\alpha} \partial_{\alpha} X^{\mu}$, the on-shell momentum $P_{\mu}$ is given by

$$
\begin{aligned}
2 \pi \alpha^{\prime} P_{\mu} & =e^{-1} g_{\mu \nu} \stackrel{\circ}{ }^{\nu}+\mathcal{B}_{\mu \nu} \partial_{\sigma} X^{\nu}+\mathcal{A}_{\mu}{ }^{M} Z_{M}, \\
\stackrel{\circ}{X}^{\mu} & :=\partial_{\tau} X^{\mu}-u \partial_{\sigma} X^{\mu},
\end{aligned}
$$

according to (3.13), and no further investigation is required.

For the internal $\left(Y^{i}, P_{i}\right)$ sector, we choose the Hamiltonian form (A23) over the Lagrangian one (A22), a choice that we will motivate at the end of this section. According to (A23), the transformations for the phase space variables $Y^{i}$ and $P_{i}$ are given by

$$
\begin{aligned}
& \delta Y^{i}=\alpha \partial_{\sigma} Y^{i}+\epsilon \hat{\mathcal{H}}^{i} \hat{N} \hat{Z}^{\hat{N}}, \\
& \delta P_{i}=\partial_{\sigma}\left[\alpha P_{i}+\frac{1}{2 \pi \alpha^{\prime}} \epsilon \hat{\mathcal{H}}_{i \hat{N}} \hat{Z}^{\hat{N}}\right],
\end{aligned}
$$

where, crucially, the last term of (A23) vanishes in the transformation of $P_{i}$, thanks to $\partial_{i} \Phi(X)=0$ for any spacetime field. This last fact implies the most important property we were after: $P_{i}$ transforms as a total $\sigma$ derivative under diffeomorphisms. ${ }^{7}$ This allows us to implement the field redefinition $2 \pi \alpha^{\prime} P_{i}=\partial_{\sigma} \tilde{Y}_{i}$ without introducing

\footnotetext{
${ }^{7}$ This also implies that the center-of-mass truncation $p_{i 0}=0$ is diffeomorphism invariant.
} 
nonlocalities in the transformations. Indeed, consistently with (A26), we can write

$$
\begin{aligned}
& \delta Y^{i}=\alpha \partial_{\sigma} Y^{i}+\epsilon \hat{\mathcal{H}}^{i} \hat{\mathcal{N}}^{\hat{N}}, \\
& \delta \tilde{Y}_{i}=\alpha \partial_{\sigma} \tilde{Y}_{i}+\epsilon \hat{\mathcal{H}}_{i \hat{N}} \hat{Z}^{\hat{N}} .
\end{aligned}
$$

At this point, $O(d, d)$ invariance may look manifest. However, despite the simple-looking form, the decomposition of the $O(d+n, d+n)$ generalized metric in terms of $O(d, d)$ covariant $n$-dimensional fields is somewhat involved, see [45]. Upon using the Kaluza-Klein decomposition (3.6), (3.7), as well as (A24) and (A25), we find, after a straightforward but tedious computation

$\hat{\mathcal{H}}^{i}{ }_{\hat{N}} \hat{Z}^{\hat{N}}=\mathcal{H}^{i}{ }_{M} D_{\sigma} Y^{M}-e^{-1} A_{\mu}^{i}\left(\partial_{\tau} X^{\mu}-u \partial_{\sigma} X^{\mu}\right)$,

$\hat{\mathcal{H}}_{i \hat{N}} \hat{Z}^{\hat{N}}=\mathcal{H}_{i M} D_{\sigma} Y^{M}-e^{-1} \tilde{A}_{\mu i}\left(\partial_{\tau} X^{\mu}-u \partial_{\sigma} X^{\mu}\right)$.

This establishes the diffeomorphism transformations of the double coordinates $Y^{M}$ in a manifestly local and $O(d, d)$ covariant form:

$\delta Y^{M}=\alpha \partial_{\sigma} Y^{M}+\epsilon\left[\mathcal{H}^{M N} D_{\sigma} Y_{N}-e^{-1} \mathcal{A}_{\mu}{ }^{M}\left(\partial_{\tau} X^{\mu}-u \partial_{\sigma} X^{\mu}\right)\right]$.

Recalling the relation (A20) between the Hamiltonian gauge parameters $(\alpha, \epsilon)$ and the $\mathfrak{d} \mathbf{i f f} \tilde{f}_{2}$ vector $\xi^{\alpha}$, it is possible to rewrite the above transformation law in a more illuminating form:

$\delta Y^{M}=\xi^{\alpha} \partial_{\alpha} Y^{M}-\xi^{\tau}\left[D_{\tau} Y^{M}-u D_{\sigma} Y^{M}-e \mathcal{H}^{M N} D_{\sigma} Y_{N}\right]$.

The above transformation law reduces to the standard one, $\delta Y^{M}=\xi^{\alpha} \partial_{\alpha} Y^{M}$, upon using the self-duality relation (3.29). However, (A30) provides the correct off-shell biff 2 transformation in the general case. To summarize, the action (3.20) is invariant under the worldsheet diffeomorphisms

$$
\begin{aligned}
\delta_{\xi} X^{\mu} & =\xi^{\alpha} \partial_{\alpha} X^{\mu}, \quad \delta_{\xi} h_{\alpha \beta}=\nabla_{\alpha} \xi_{\beta}+\nabla_{\beta} \xi_{\alpha} \\
\delta_{\xi} Y^{M} & =\xi^{\alpha} \partial_{\alpha} Y^{M}-\xi^{\tau} \mathfrak{D}^{M}
\end{aligned}
$$

where we defined the "self-duality vector"

$$
\mathfrak{D}^{M}:=D_{\tau} Y^{M}-u D_{\sigma} Y^{M}-e \mathcal{H}^{M N} D_{\sigma} Y_{N},
$$

and we recall that the transformation law (A3) of $e$ and $u$ is just determined by their definition

$$
e=\frac{\sqrt{-h}}{h_{\sigma \sigma}}, \quad u=\frac{h_{\tau \sigma}}{h_{\sigma \sigma}} .
$$

Invariance of (3.20) under (A31) is assured by the general reasoning leading to (A30), but it can also be checked directly by using

$$
\begin{aligned}
\delta_{\xi}\left(D_{\alpha} Y^{M}\right)= & \mathcal{L}_{\xi}\left(D_{\alpha} Y^{M}\right)-\partial_{\alpha}\left(\xi^{\tau} \mathfrak{D}^{M}\right), \\
\delta_{\xi} \mathfrak{D}^{M}= & \xi^{\alpha} \partial_{\alpha} \mathfrak{D}^{M}-\xi^{\tau}\left[\partial_{\tau} \mathfrak{D}^{M}-u \partial_{\sigma} \mathfrak{D}^{M}\right. \\
& \left.-e \mathcal{H}^{M N} \partial_{\sigma} \mathfrak{D}_{N}\right] .
\end{aligned}
$$

The variations (A34) can also be used to check that the algebra of diffeomorphisms closes off-shell, even with the extra term, according to the usual Lie bracket:

$$
\left[\delta_{\xi_{2}}, \delta_{\xi_{1}}\right]=\delta_{\xi_{12}}, \quad \xi_{12}^{\alpha}=\xi_{1}^{\beta} \partial_{\beta} \xi_{2}^{\alpha}-\xi_{2}^{\beta} \partial_{\beta} \xi_{1}^{\alpha} .
$$

The zero mode shift symmetry (3.22)

$$
\delta_{\Xi} Y^{M}(\sigma, \tau)=\Xi^{M}(\tau), \quad \delta_{\Xi} X^{\mu}(\sigma, \tau)=0, \quad \delta_{\Xi} h_{\alpha \beta}(\sigma, \tau)=0,
$$

commutes with diffeomorphisms: $\left[\delta_{\xi}, \delta_{\Xi}\right]=0$. However, the gauge fixing condition (3.27) is not $\boldsymbol{D} \mathbf{i f f}_{2}$ invariant offshell. The easiest way to see this is to notice that (3.27) can be written as

$$
\frac{1}{2 \pi} \int_{0}^{2 \pi} d \sigma \mathfrak{D}^{M}=0
$$

and its variation under a diffeomorphism is given by

$$
\begin{aligned}
& \delta_{\xi}\left(\int_{0}^{2 \pi} d \sigma \mathfrak{D}^{M}\right) \\
& \quad=\int_{0}^{2 \pi} d \sigma\left[\xi^{\sigma} \partial_{\sigma} \mathfrak{D}^{M}+\xi^{\tau}\left(u \partial_{\sigma} \mathfrak{D}^{M}+e \mathcal{H}^{M N} \partial_{\sigma} \mathfrak{D}_{N}\right)\right],
\end{aligned}
$$

that vanishes only on-shell, by noting that the $Y$ field equation (3.28) is just $\partial_{\sigma} \mathfrak{D}^{M}=0$. This is not in contradiction with our claim, namely that the string theory described by (3.20) is classically equivalent to the truncated sector of the original sigma model, meaning that the equivalence holds at the level of the space of classical solutions.

\section{Energy-momentum tensor and conformal symmetry}

We will now derive consequences of diffeomorphism invariance such as energy-momentum conservation. The diffeomorphism invariance of the action (3.20) can be expressed as

$$
\int d^{2} \sigma\left[\frac{\delta S}{\delta \varphi^{A}} \delta_{\xi} \varphi^{A}+\frac{\delta S}{\delta e} \delta_{\xi} e+\frac{\delta S}{\delta u} \delta_{\xi} u\right]=0,
$$


where we grouped the "matter fields" as $\varphi^{A}:=\left(X^{\mu}, Y^{M}\right)$. By evaluating (A39) for on-shell configurations of the matter fields, i.e., $\frac{\delta S}{\delta \varphi^{A}}=0$, one obtains

$$
\begin{aligned}
& \int d^{2} \sigma\left[\mathcal{H}\left(\partial_{\tau} \epsilon-u \partial_{\sigma} \epsilon-e \partial_{\sigma} \alpha+\partial_{\sigma} e \alpha\right)\right. \\
& \left.+\mathcal{N}\left(\partial_{\tau} \alpha-u \partial_{\sigma} \alpha-e \partial_{\sigma} \epsilon+\partial_{\sigma} e \epsilon\right)\right]=0
\end{aligned}
$$

where we used (A14). The functions $\mathcal{H}=-\frac{\delta S}{\delta e}$ and $\mathcal{N}=-\frac{\delta S}{\delta u}$ are given by

$$
\begin{aligned}
\mathcal{H}= & \frac{1}{4 \pi \alpha^{\prime}}\left[e^{-2} g_{\mu \nu} \stackrel{\circ}{X^{\mu}} \stackrel{\circ}{ }^{\nu}+g_{\mu \nu} \partial_{\sigma} X^{\mu} \partial_{\sigma} X^{\nu}\right. \\
& \left.+\mathcal{H}_{M N} D_{\sigma} Y^{M} D_{\sigma} Y^{N}\right], \\
\mathcal{N}= & \frac{1}{4 \pi \alpha^{\prime}}\left[2 e^{-1} g_{\mu \nu} \stackrel{\circ}{ }^{\mu} \partial_{\sigma} X^{\nu}+D_{\sigma} Y^{M} D_{\sigma} Y_{M}\right],
\end{aligned}
$$

where $\stackrel{\circ}{X^{\mu}}=\partial_{\tau} X^{\mu}-u \partial_{\sigma} X^{\mu}$. Since (A40) holds for arbitrary $\epsilon$ and $\alpha$, we obtain the energy-momentum conservation law in arbitrary gauge:

$$
\begin{aligned}
\partial_{\tau} \mathcal{H} & =\partial_{\sigma}(e \mathcal{N}+u \mathcal{H})+\partial_{\sigma} u \mathcal{H}+\partial_{\sigma} e \mathcal{N}, \\
\partial_{\tau} \mathcal{N} & =\partial_{\sigma}(e \mathcal{H}+u \mathcal{N})+\partial_{\sigma} u \mathcal{N}+\partial_{\sigma} e \mathcal{H} .
\end{aligned}
$$

Since diffeomorphism invariance can be used to fix the metric components $e$ and $u$, one is mostly interested in studying the model (3.20) in conformal gauge, that corresponds to $e=1$ and $u=0$. The action (3.20) then reduces to

$$
\begin{aligned}
S_{\text {c.g. }}= & -\frac{1}{4 \pi \alpha^{\prime}} \int d^{2} \sigma\left[g_{\mu \nu} \partial^{\alpha} X^{\mu} \partial_{\alpha} X^{\nu}\right. \\
& \left.+\epsilon^{\alpha \beta}\left(B_{\mu \nu} \partial_{\alpha} X^{\mu} \partial_{\beta} X^{\nu}-\mathcal{A}_{\mu}{ }^{M} D_{\alpha} Y_{M} \partial_{\beta} X^{\mu}\right)\right] \\
& +\frac{1}{4 \pi \alpha^{\prime}} \int d^{2} \sigma\left[D_{\sigma} Y^{M} D_{\tau} Y_{M}-\mathcal{H}_{M N} D_{\sigma} Y^{M} D_{\sigma} Y^{N}\right] .
\end{aligned}
$$

This has to be supplemented with the Virasoro constraints $\mathcal{H}=0, \mathcal{N}=0$. In conformal gauge it is useful to introduce light-cone worldsheet coordinates $\sigma^{ \pm}:=\tau \pm \sigma$, for which one has

$$
\begin{aligned}
\partial_{ \pm} & =\frac{1}{2}\left(\partial_{\tau} \pm \partial_{\sigma}\right), \quad \eta_{+-}=-\frac{1}{2}, \\
\eta^{+-} & =-2, \quad \epsilon^{+-}=2, \quad \epsilon_{+-}=-\frac{1}{2} .
\end{aligned}
$$

The Virasoro constraints can then be expressed in the more familiar form $T_{ \pm \pm}=0$, defined by

$$
\begin{aligned}
T_{++} & :=\pi\left(\mathcal{H}_{\text {c.g. }}+\mathcal{N}_{\text {c.g. }}\right) \\
& =\frac{1}{\alpha^{\prime}}\left(g_{\mu \nu} \partial_{+} X^{\mu} \partial_{+} X^{\nu}+\frac{1}{2} \Pi_{M N}^{+} D_{\sigma} Y^{M} D_{\sigma} Y^{N}\right), \\
T_{--} & :=\pi\left(\mathcal{H}_{\text {c.g. }}-\mathcal{N}_{\text {c.g. }}\right) \\
& =\frac{1}{\alpha^{\prime}}\left(g_{\mu \nu} \partial_{-} X^{\mu} \partial_{-} X^{\nu}-\frac{1}{2} \Pi_{M N}^{-} D_{\sigma} Y^{M} D_{\sigma} Y^{N}\right),
\end{aligned}
$$

where the $O(d, d)$ projectors are as in (A11) and $D_{\sigma}=D_{+}-D_{-}$.

Conformal symmetry of the gauge fixed action (A43) is easily established as the global remnant of diffeomorphisms that preserve the conformal gauge choice. From the transformation law (A14) one has the conditions

$\left.\delta e\right|_{\mathrm{conf}}=\partial_{\tau} \epsilon-\partial_{\sigma} \alpha=0,\left.\quad \delta u\right|_{\mathrm{conf}}=\partial_{\tau} \alpha-\partial_{\sigma} \epsilon=0$

to preserve the conformal gauge. Since in this gauge Hamiltonian and Lagrangian parameters coincide, $\xi^{\alpha}=(\epsilon, \alpha)$, one can easily see that the conditions (A46) are equivalent to the usual analyticity

$$
\partial_{-} \xi^{+}=0, \quad \partial_{+} \xi^{-}=0 .
$$

The diffeomorphism transformations (A31) give directly the conformal transformations leaving (A43) invariant:

$$
\begin{aligned}
\delta_{\text {conf }} X^{\mu}= & \xi^{+} \partial_{+} X^{\mu}+\xi^{-} \partial_{-} X^{\mu}, \\
\delta_{\text {conf }} Y^{M}= & \xi^{+} \partial_{+} Y^{M}+\xi^{-} \partial_{-} Y^{M}-\left(\xi^{+}+\xi^{-}\right) \\
& \times\left[\Pi_{-}^{M}{ }_{N} D_{+} Y^{N}+\Pi_{+}^{M} D_{-} Y^{N}\right],
\end{aligned}
$$

for analytic parameters $\xi^{+}\left(\sigma^{+}\right), \xi^{-}\left(\sigma^{-}\right)$. Moreover, the usual $\mathrm{Diff}_{2}$ algebra (A35) ensures that conformal transformations form two commuting copies of the classical Virasoro (Witt) algebra.

As a final remark, we notice that the conservation law (A42) reduces in conformal gauge to analyticity of the energy-momentum tensor:

$$
\partial_{-} T_{++}=0, \quad \partial_{+} T_{--}=0,
$$

despite the nonstandard contributions from the $Y$ sector. In fact, we shall also notice that the self-duality relation (3.29), that is $\mathfrak{D}^{M}=0$, can be written in conformal gauge as

$$
\Pi_{M N}^{ \pm} D_{\mp} Y^{N}=0 .
$$

If (A50) is imposed, both the conformal transformations of $Y^{M}$ and the $Y$ contribution to the stress-energy tensor assume the standard form 


$$
\begin{aligned}
\delta_{\text {conf. }} Y^{M} & =\xi^{+} \partial_{+} Y^{M}+\xi^{-} \partial_{-} Y^{M} \\
T_{ \pm \pm} & =\frac{1}{\alpha^{\prime}}\left(g_{\mu \nu} \partial_{ \pm} X^{\mu} \partial_{ \pm} X^{\nu}+\frac{1}{2} \mathcal{H}_{M N} D_{ \pm} Y^{M} D_{ \pm} Y^{N}\right)
\end{aligned}
$$

but one should always keep in mind that (A50) is not a variational equation.

\section{APPENDIX B: CANCELLATION OF GRAVITATIONAL ANOMALIES}

In this Appendix we verify explicitly that for the model carrying $d$ left-moving and $d$ right-moving chiral bosons the gravitational anomalies cancel. To this end we compute the one-loop effective action for the gravitational field and establish that gravitational anomalies can be canceled without spoiling $O(d, d)$ invariance. For simplicity we consider the theory defined by the action (3.20) for the case of vanishing gauge fields, $\mathcal{A}_{\mu}{ }^{M}=0$, and constant generalized metric: $\partial_{\mu} \mathcal{H}_{M N}=0$. The $Y$-sector decouples from the $X$-sector and reduces to the sum of left and right Floreanini-Jackiw actions coupled to gravity [46-48]:

$$
\begin{aligned}
S\left[Y, e_{ \pm}\right]= & \frac{1}{4 \pi \alpha^{\prime}} \int d^{2} \sigma\left[\partial_{\sigma} Y_{L}^{M}\left(\partial_{\tau}-e_{+} \partial_{\sigma}\right) Y_{L M}\right. \\
& \left.+\partial_{\sigma} Y_{R}^{M}\left(\partial_{\tau}-e_{-} \partial_{\sigma}\right) Y_{R M}\right]
\end{aligned}
$$

where we used the $O(d, d)$ projectors to define

$$
Y_{L}^{M}:=\Pi_{+N}^{M} Y^{N}, \quad Y_{R}^{M}:=\Pi_{-N}^{M} Y^{N}
$$

and introduced $e_{ \pm}:=u \pm e$. The action (B1) is invariant under two-dimensional diffeomorphisms acting as

$$
\begin{aligned}
\delta_{\varepsilon} Y_{L}^{M} & =\varepsilon_{+} \partial_{\sigma} Y_{L}^{M}, \quad \delta_{\varepsilon} Y_{R}^{M}=\varepsilon_{-} \partial_{\sigma} Y_{R}^{M}, \\
\delta_{\varepsilon} e_{ \pm} & =\partial_{\tau} \varepsilon_{ \pm}-e_{ \pm} \partial_{\sigma} \varepsilon_{ \pm}+\varepsilon_{ \pm} \partial_{\sigma} e_{ \pm},
\end{aligned}
$$

where the parameters $\varepsilon_{ \pm}=\alpha \pm \epsilon$ are given by

$$
\varepsilon_{ \pm}=\xi^{\sigma}+e_{ \pm} \xi^{\tau}
$$

in terms of the usual vector field $\xi^{\alpha}$. The action is also invariant under two separate zero-mode local symmetries:

$$
\delta_{\Xi} Y_{L}^{M}(\sigma, \tau)=\Xi_{L}^{M}(\tau), \quad \delta_{\Xi} Y_{R}^{M}(\sigma, \tau)=\Xi_{R}^{M}(\tau) .
$$

In order to compute the one-loop effective action for the gravitational field we start by shifting the gravity fields as

$$
e_{+}=1+\varphi_{+}, \quad e_{-}=-1-\varphi_{-},
$$

so that $\varphi_{ \pm}=0$ in conformal gauge. The action (B1) then splits into a quadratic part and an interaction term, allowing for a well-defined perturbative treatment:

$$
\begin{aligned}
S\left[Y, \varphi_{ \pm}\right]= & \frac{1}{4 \pi \alpha^{\prime}} \int d^{2} \sigma\left[\partial_{\sigma} Y_{L}^{M}\left(\partial_{\tau}-\partial_{\sigma}\right) Y_{L M}\right. \\
& \left.+\partial_{\sigma} Y_{R}^{M}\left(\partial_{\tau}+\partial_{\sigma}\right) Y_{R M}\right] \\
& +\frac{1}{4 \pi \alpha^{\prime}} \int d^{2} \sigma\left[\varphi_{-} \partial_{\sigma} Y_{R} \cdot \partial_{\sigma} Y_{R}\right. \\
& \left.-\varphi_{+} \partial_{\sigma} Y_{L} \cdot \partial_{\sigma} Y_{L}\right] \\
= & S_{L}\left[Y_{L}, \varphi_{+}\right]+S_{R}\left[Y_{R}, \varphi_{-}\right],
\end{aligned}
$$

where the dot denotes contraction of $O(d, d)$ indices with $\eta_{M N}$. Since the action is the sum of independent left and right terms, the path integral ${ }^{8}$ factorizes:

$e^{i W\left[\varphi_{ \pm}\right]}:=Z^{-1} \int D Y_{L} D Y_{R} e^{i S\left[Y, \varphi_{ \pm}\right]}=e^{i W_{L}\left[\varphi_{+}\right]} e^{i W_{R}\left[\varphi_{-}\right]}$.

We will thus focus on the left part of the effective action $W_{L}\left[\varphi_{+}\right]$, that can be written as the quantum average

$e^{i W_{L}\left[\varphi_{+}\right]}=Z_{L}^{-1} \int D Y_{L} e^{i S_{L}\left[Y_{L}, \varphi_{+}\right]}=:\left\langle e^{-\frac{i}{4 \pi \alpha^{\prime}} \int d^{2} \sigma \varphi_{+} \partial_{\sigma} Y_{L} \cdot \partial_{\sigma} Y_{L}}\right\rangle$,

and just present the result for $W_{R}\left[\varphi_{-}\right]$.

Due to the zero-mode symmetry (B5), the kinetic operator $\partial_{\sigma}\left(\partial_{\tau}-\partial_{\sigma}\right)$ is not invertible. Gauge fixing (B5) with appropriate boundary conditions at asymptotic times [46] yields a trivial path integral for the zero-mode $Y_{L 0}^{M}(\tau)$. The above path integral is thus understood as $\int D \bar{Y}_{L}$ over the nonzero mode part of $Y_{L}^{M}$ only:

$$
\bar{Y}_{L}^{M}(\sigma, \tau):=Y_{L}^{M}(\sigma, \tau)-Y_{L 0}^{M}(\tau)
$$

whose propagator is well defined and given by

$$
\left\langle\bar{Y}_{L}^{M}(x) \bar{Y}_{L}^{N}(y)\right\rangle=-4 \pi \alpha^{\prime} i \Pi_{+}^{M N} \int \frac{\left[d^{2} k\right]}{(2 \pi)^{2}} e^{i k \cdot(x-y)} \frac{k_{+}}{k_{1}} \frac{1}{k^{2}-i \epsilon},
$$

where the momentum $k_{\alpha}:=(\omega, n)$, with discrete $n \in \mathbb{Z}$ in the $\sigma$ direction, and the "integration" measure is defined by

$$
\int\left[d^{2} k\right]:=\int_{-\infty}^{+\infty} d \omega \sum_{n \neq 0}
$$

We have also changed notation by denoting the worldsheet coordinates as $x^{\alpha}=(\tau, \sigma)$, in order not to confuse $\sigma^{\alpha}$ with the spatial component $\sigma$.

Equipped with the propagator (B11) we can compute $W_{L}\left[\varphi_{+}\right]$up to quadratic order:

\footnotetext{
${ }^{8} \mathrm{We}$ fix the normalization $Z$ to be the free $Y$-path integral, so that $W[0]=0$.
} 


$$
\begin{aligned}
W_{L}\left[\varphi_{+}\right]= & i d \Delta \int d^{2} x \varphi_{+}(x)-i d \int d^{2} x \\
& \times \int \frac{d^{2} y}{(2 \pi)^{2}} \frac{d^{2} p}{(2 \pi)^{2}} e^{i p \cdot(x-y)} \varphi_{+}(x) \varphi_{+}(y) I(p),
\end{aligned}
$$

with the tadpole $\Delta$ and the bubble diagram $I(p)$ defined by

$$
\begin{aligned}
\Delta & :=\int \frac{\left[d^{2} k\right]}{(2 \pi)^{2}} \frac{k_{1} k_{+}}{k^{2}-i \epsilon}, \\
I(p) & :=\int\left[d^{2} k\right] \frac{k_{1} k_{+}}{k^{2}-i \epsilon} \frac{\left(p_{1}+k_{1}\right)\left(p_{+}+k_{+}\right)}{(p+k)^{2}-i \epsilon} .
\end{aligned}
$$

We regulate the frequency integrals [see (B12)] by inserting a factor of $e^{i \epsilon^{\prime} \omega}$. This is sufficient to make $I(p)$ finite, while a divergent contribution has still to be subtracted from the zero-point energy $\Delta$. Although the regulator manifestly breaks Lorentz symmetry, we will be only interested in the nonlocal part of the effective action, that is not affected by changing the regularization scheme. The finite results for the above diagrams are

$$
\Delta=-\frac{i}{48 \pi}, \quad I(p)=-\frac{i \pi}{24} \frac{p_{1}^{3}-p_{1}}{p_{-}}
$$

which allows us to write the effective action as

$$
\begin{aligned}
& W_{L}\left[\varphi_{+}\right] \\
& =\frac{d}{48 \pi} \int d^{2} x \varphi_{+}+\frac{d}{96 \pi} \int d^{2} x \varphi_{+}\left(\frac{\partial_{1}^{3}+\partial_{1}}{\partial_{-}}\right) \varphi_{+}+\mathcal{O}\left(\varphi_{+}^{3}\right) \\
& =\frac{d}{96 \pi} \int d^{2} x \varphi_{+}\left(\frac{\partial_{+}^{3}+\partial_{+}}{\partial_{-}}\right) \varphi_{+}+\text {local terms }+\mathcal{O}\left(\varphi_{+}^{3}\right) .
\end{aligned}
$$

Genuine anomalies are the ones that cannot be canceled by adding local counterterms (that reflect different regularizations) to the effective action. That is why we only need to focus on the nonlocal part of $W_{L}$ above.

We can now compute the gauge transformation of the effective action. Using the transformation law

$$
\delta_{\varepsilon} \varphi_{+}=2 \partial_{-} \varepsilon_{+}-\varphi_{+} \partial_{1} \varepsilon_{+}+\varepsilon_{+} \partial_{1} \varphi_{+},
$$

it is easy to see that the anomalous variation of (B16) is given by

$$
\delta_{\varepsilon} W_{L}^{\text {n.l. }}\left[\varphi_{+}\right]=-\frac{d}{24 \pi} \int d^{2} x \varepsilon_{+}\left(\partial_{+}^{3}+\partial_{+}\right) \varphi_{+}+\mathcal{O}\left(\varphi_{+}^{2}\right) .
$$

The term $\varepsilon_{+} \partial_{+} \varphi_{+}$can be canceled by adding a local counterterm proportional to $\varphi_{+}-\frac{1}{2} \varphi_{+}^{2}$, but the term cubic in $\partial_{+}$cannot be canceled and represents the genuine gravitational anomaly (to lowest order in $\varphi_{+}$) of the chiral bosons $Y_{L}^{M}$ on the cylinder. The anomaly is the same obtained on the plane from $d$ left-moving FloreaniniJackiw bosons $[47,49,50]$. This ensures that adding the contribution $W_{R}\left[\varphi_{-}\right]$allows us to cancel the gravitational anomaly completely.

To be more explicit, adding the contribution from the right-moving fields $Y_{R}^{M}$ one obtains the full nonlocal contribution:

$$
W^{\mathrm{n} . \mathrm{l}}\left[\varphi_{ \pm}\right]=\frac{d}{96 \pi} \int d^{2} x\left\{\varphi_{+}\left(\frac{\partial_{+}^{3}+\partial_{+}}{\partial_{-}}\right) \varphi_{+}+\varphi_{-}\left(\frac{\partial_{-}^{3}+\partial_{-}}{\partial_{+}}\right) \varphi_{-}\right\}+\mathcal{O}\left(\varphi_{ \pm}^{3}\right)
$$

The gravitational anomaly of the above expression can indeed be canceled by adding a local counterterm that involves the third degree of freedom of the worldsheet metric: the conformal factor $\Omega$. Denoting the deviation of $\Omega$ from its flat space value by $\phi:=\Omega-1$ one can add to (B19) a local counterterm $\Delta W\left[\varphi_{ \pm}, \phi\right]$ and define the effective action as

$$
\begin{aligned}
W_{\mathrm{eff}}\left[\varphi_{ \pm}, \phi\right]= & \frac{d}{96 \pi} \int d^{2} x\left\{\varphi_{+} \frac{\partial_{+}^{3}}{\partial_{-}} \varphi_{+}+\varphi_{-} \frac{\partial_{-}^{3}}{\partial_{+}} \varphi_{-}+2 \varphi_{+} \partial_{+} \partial_{-} \varphi_{-}-4 \phi\left(\partial_{+}^{2} \varphi_{+}+\partial_{-}^{2} \varphi_{-}\right)+4 \phi \partial_{+} \partial_{-} \phi\right\} \\
& +\frac{d}{96 \pi} \int d^{2} x\left\{\varphi_{+} \frac{\partial_{+}}{\partial_{-}} \varphi_{+}+2 \varphi_{+}-\varphi_{+}^{2}+\varphi_{-} \frac{\partial_{-}}{\partial_{+}} \varphi_{-}+2 \varphi_{-}-\varphi_{-}^{2}\right\}+\mathcal{O}\left(\text { fields }^{3}\right) .
\end{aligned}
$$

One can check that the above effective action is invariant under diffeomorphisms (to lowest order in the fields, since we are considering only the quadratic part of $W_{\text {eff }}$ ) with transformations 


$$
\begin{aligned}
\delta_{\varepsilon} \varphi_{+}= & 2 \partial_{-} \varepsilon_{+}-\varphi_{+} \partial_{1} \varepsilon_{+}+\varepsilon_{+} \partial_{1} \varphi_{+}, \\
\delta_{\varepsilon} \varphi_{-}= & -2 \partial_{+} \varepsilon_{-}-\varphi_{-} \partial_{1} \varepsilon_{-}+\varepsilon_{-} \partial_{1} \varphi_{-}, \\
\delta \phi= & \partial_{+} \varepsilon_{+}-\partial_{-} \varepsilon_{-}+\frac{1}{2} \partial_{+}\left[\varphi_{+}\left(\varepsilon_{-}-\varepsilon_{+}\right)+2 \phi \varepsilon_{+}\right] \\
& +\frac{1}{2} \partial_{-}\left[\varphi_{-}\left(\varepsilon_{-}-\varepsilon_{+}\right)-2 \phi \varepsilon_{-}\right]+\mathcal{O}\left(\text { field }^{2}\right) .
\end{aligned}
$$

Let us mention that the last line in (B20) is invariant by itself and the tadpoles (linear terms) in $\varphi_{ \pm}$just reflect the nonzero Casimir energy on the cylinder, i.e., $\left\langle T_{ \pm \pm}\right\rangle \neq 0$.

As it happens for ordinary scalars, the price to pay to restore diffeomorphism invariance is the breakdown of Weyl symmetry. While $\varphi_{ \pm}$are exactly Weyl invariant, $\delta_{\omega} \phi=2 \omega+\mathcal{O}(\phi)$ and one readily obtains

$$
\begin{aligned}
\delta_{\omega} W_{\text {eff }}\left[\varphi_{ \pm}, \phi\right]= & \frac{d}{12 \pi} \int d^{2} x \omega\left(2 \partial_{+} \partial_{-} \phi-\partial_{+}^{2} \varphi_{+}-\partial_{-}^{2} \varphi_{-}\right) \\
& +\mathcal{O}\left(\text { field }^{2}\right) .
\end{aligned}
$$

To lowest order in the fields this is

$$
\delta_{\omega} W_{\mathrm{eff}}\left[\varphi_{ \pm}, \phi\right]=\frac{d}{24 \pi} \int d^{2} x \sqrt{-h} \omega R
$$

thus yielding the trace anomaly

$$
\left\langle T_{\alpha}^{\alpha}\right\rangle=-\frac{d}{12} R
$$

This confirms that the internal $Y^{M}$ sector just contributes to the trace anomaly with $d$ units of both left and right central charge. Indeed, including the $n$ external ordinary bosons $X^{\mu}$, one has the requirement $n+d=26$ for criticality.
[1] A. A. Tseytlin, Duality symmetric formulation of string world sheet dynamics, Phys. Lett. B 242, 163 (1990).

[2] A. A. Tseytlin, Duality symmetric closed string theory and interacting chiral scalars, Nucl. Phys. B350, 395 (1991).

[3] G. Veneziano, Scale factor duality for classical and quantum strings, Phys. Lett. B 265, 287 (1991).

[4] M. Gasperini and G. Veneziano, $\mathrm{O}(\mathrm{d}, \mathrm{d})$ covariant string cosmology, Phys. Lett. B 277, 256 (1992).

[5] T. Kugo and B. Zwiebach, Target space duality as a symmetry of string field theory, Prog. Theor. Phys. 87, 801 (1992).

[6] J. Maharana and J. H. Schwarz, Noncompact symmetries in string theory, Nucl. Phys. B390, 3 (1993).

[7] W. Siegel, Superspace duality in low-energy superstrings, Phys. Rev. D 48, 2826 (1993).

[8] C. M. Hull, Doubled geometry and T-folds, J. High Energy Phys. 07 (2007) 080.

[9] C. M. Hull, Global aspects of T-duality, gauged sigma models and T-folds, J. High Energy Phys. 10 (2007) 057.

[10] D. S. Berman and N.B. Copland, The string partition function in Hull's doubled formalism, Phys. Lett. B 649, 325 (2007).

[11] D. S. Berman, N. B. Copland, and D. C. Thompson, Background field equations for the duality symmetric string, Nucl. Phys. B791, 175 (2008).

[12] D. S. Berman and D.C. Thompson, Duality symmetric strings, dilatons and $\mathrm{O}(\mathrm{d}, \mathrm{d})$ effective actions, Phys. Lett. B 662, 279 (2008).

[13] J. Maharana, Duality symmetry of string theory: A worldsheet perspective, Phys. Lett. B 695, 370 (2011).
[14] C. D. A. Blair, E. Malek, and A. J. Routh, An $O(D, D)$ invariant Hamiltonian action for the superstring, Classical Quantum Gravity 31, 205011 (2014).

[15] L. De Angelis, G. G. Sj, R. Marotta, and F. Pezzella, Comparing double string theory actions, J. High Energy Phys. 04 (2014) 171.

[16] M. Hatsuda, K. Kamimura, and W. Siegel, Type II chiral affine Lie algebras and string actions in doubled space, J. High Energy Phys. 09 (2015) 113.

[17] I. Bakas, D. Lust, and E. Plauschinn, Towards a world-sheet description of doubled geometry in string theory, Fortschr. Phys. 64, 730 (2016).

[18] S. Driezen, A. Sevrin, and D. C. Thompson, Aspects of the doubled worldsheet, J. High Energy Phys. 12 (2016) 082.

[19] C. Eloy, O. Hohm, and H. Samtleben, Green-Schwarz Mechanism for String Dualities, Phys. Rev. Lett. 124, 091601 (2020).

[20] C. Eloy, O. Hohm, and H. Samtleben, Duality invariance and higher derivatives, Phys. Rev. D 101, 126018 (2020).

[21] O. Hohm, W. Siegel, and B. Zwiebach, Doubled $\alpha^{\prime}$-geometry, J. High Energy Phys. 02 (2014) 065.

[22] O. Hohm and B. Zwiebach, Green-Schwarz mechanism and $\alpha^{\prime}$-deformed Courant brackets, J. High Energy Phys. 01 (2015) 012.

[23] O. Hohm and B. Zwiebach, Double field theory at order $\alpha^{\prime}$, J. High Energy Phys. 11 (2014) 075.

[24] D. Marques and C. A. Nunez, T-duality and $\alpha^{\prime}$-corrections, J. High Energy Phys. 10 (2015) 084.

[25] O. Hohm, Background Independence and Duality Invariance in String Theory, Phys. Rev. Lett. 118, 131601 (2017). 
[26] W. H. Baron, J. J. Fernandez-Melgarejo, D. Marques, and C. Nunez, The odd story of $\alpha^{\prime}$-corrections, J. High Energy Phys. 04 (2017) 078.

[27] J.H. Schwarz and A. Sen, Duality symmetries of 4-D heterotic strings, Phys. Lett. B 312, 105 (1993).

[28] C. D. A. Blair, Doubled strings, negative strings and null waves, J. High Energy Phys. 11 (2016) 042.

[29] C. M. Hull and E. Witten, Supersymmetric sigma models and the heterotic string, Phys. Lett. 160B, 398 (1985).

[30] A. Sen, Local gauge and Lorentz invariance of the heterotic string theory, Phys. Lett. 166B, 300 (1986).

[31] L. Alvarez-Gaume and E. Witten, Gravitational anomalies, Nucl. Phys. B234, 269 (1984).

[32] R. Roiban and A. A. Tseytlin, On duality symmetry in perturbative quantum theory, J. High Energy Phys. 10 (2012) 099.

[33] B. Hoare, N. Levine, and A. A. Tseytlin, On the massless tree-level S-matrix in 2d sigma models, J. Phys. A 52, 144005 (2019).

[34] M. B. Green and J. H. Schwarz, Anomaly cancellation in supersymmetric $D=10$ gauge theory and superstring theory, Phys. Lett. 149B, 117 (1984).

[35] R. Floreanini and R. Jackiw, Selfdual Fields as Charge Density Solitons, Phys. Rev. Lett. 59, 1873 (1987).

[36] W. Siegel, Two Vierbein formalism for string inspired axionic gravity, Phys. Rev. D 47, 5453 (1993).

[37] O. Hohm, C. Hull, and B. Zwiebach, Generalized metric formulation of double field theory, J. High Energy Phys. 08 (2010) 008.

[38] C. G. Callan, Jr. and L. Thorlacius, Sigma models and string theory, in Theoretical Advanced Study Institute in Elementary Particle Physics: Particles, Strings and
Supernovae (TASI 88) (Proceedings, Particles, strings and supernovae, Providence, RI, United States, 1988), Vol. 2, pp. 795-878.

[39] A. Bilal, Lectures on anomalies, arXiv:0802.0634.

[40] O. Hohm and B. Zwiebach, Duality invariant cosmology to all orders in $\alpha^{\prime}$, Phys. Rev. D 100, 126011 (2019).

[41] A. Sen, $\mathrm{O}(\mathrm{d}) \mathrm{x} \mathrm{O}(\mathrm{d})$ symmetry of the space of cosmological solutions in string theory, scale factor duality and two-dimensional black holes, Phys. Lett. B 271, 295 (1991).

[42] K. A. Meissner, Symmetries of higher order string gravity actions, Phys. Lett. B 392, 298 (1997).

[43] C. Hull and B. Zwiebach, Double field theory, J. High Energy Phys. 09 (2009) 099.

[44] P. K. Townsend, Manifestly Lorentz Invariant Chiral Boson Action, Phys. Rev. Lett. 124, 101604 (2020).

[45] O. Hohm, A. Sen, and B. Zwiebach, Heterotic effective action and duality symmetries revisited, J. High Energy Phys. 02 (2015) 079.

[46] M. Henneaux and C. Teitelboim, Consistent quantum mechanics of chiral $\mathrm{p}$ forms, in Second Meeting on Quantum Mechanics of Fundamental Systems (CECS) (1987), pp. 79-112 [arXiv:0802.0634].

[47] J. Sonnenschein and C. bosons, Chiral bosons, Nucl. Phys. B309, 752 (1988).

[48] F. Bastianelli and P. van Nieuwenhuizen, Chiral bosons coupled to supergravity, Phys. Lett. B 217, 98 (1989).

[49] F. Bastianelli, On chiral bosonization, Phys. Lett. B 254, 427 (1991).

[50] S. Giaccari and P. Menotti, Consistent gravitational anomalies for chiral bosons, Phys. Rev. D 79, 065015 (2009). 